\title{
Analysis of an augmented mixed-FEM for the Navier-Stokes problem*
}

\author{
Jessika Camaño† Ricardo Oyarzúa $a^{\ddagger}$ and Giordano Tierra ${ }^{\S}$
}

\begin{abstract}
In this paper we propose and analyze a new augmented mixed finite element method for the Navier-Stokes problem. Our approach is based on the introduction of a "nonlinearpseudostress" tensor linking the pseudostress tensor with the convective term, which leads to a mixed formulation with the nonlinear-pseudostress tensor and the velocity as the main unknowns of the system. Further variables of interest, such as the fluid pressure, the fluid vorticity and the fluid velocity gradient, can be easily approximated as a simple postprocess of the finite element solutions with the same rate of convergence. The resulting mixed formulation is augmented by introducing Galerkin least-squares type terms arising from the constitutive and equilibrium equations of the Navier-Stokes equations and from the Dirichlet boundary condition, which are multiplied by stabilization parameters that are chosen in such a way that the resulting continuous formulation becomes well-posed. Then, the classical Banach's fixed point Theorem and Lax-Milgram's Lemma are applied to prove well-posedness of the continuous problem. Similarly, we establish well-posedness and the corresponding Cea's estimate of the associated Galerkin scheme considering any conforming finite element subspace for each unknown. In particular, the associated Galerkin scheme can be defined by employing Raviart-Thomas elements of degree $k$ for the nonlinear-pseudostress tensor, and continuous piecewise polynomial elements of degree $k+1$ for the velocity, which leads to an optimal convergent scheme. In addition, we provide two iterative methods to solve the corresponding nonlinear system of equations and analyze their convergence. Finally, several numerical results illustrating the good performance of the method are provided.
\end{abstract}

Key words: Navier-Stokes, mixed finite element method, augmented formulation, RaviartThomas elements

Mathematics Subject Classifications (1991): 65N15, 65N30, 76D05, 76M10

${ }^{*}$ This research was partially supported by CONICYT-Chile through project Inserción de Capital Humano Avanzado en la Academia 79130048; project Fondecyt 11121347, project Anillo ACT1118 (ANANUM); by DIUBB project 120808 GI/EF; and by Ministry of Education, Youth and Sports of the Czech Republic through the ERCCZ project LL1202.

†Departamento de Matemática y Física Aplicadas, Universidad Católica de la Santísima Concepción, Casilla 297, Concepción, Chile, and CI²MA, Universidad de Concepción, Casilla 160-C, Concepción, Chile, email: jecamano@ucsc.cl

${ }^{\ddagger}$ Departamento de Matemática, Universidad del Bío-Bío, Casilla 5-C, Concepción, Chile, and CI²MA, Universidad de Concepción, Casilla 160-C, Concepción, Chile, email: royarzua@ubiobio.cl

${ }^{\S}$ Mathematical Institute, Faculty of Mathematics and Physics, Charles University, Prague 8, 186 75, Czech Republic, email: gtierra@karlin.mff.cuni.cz 


\section{Introduction}

This paper is concerned with the numerical approximation of the incompressible Navier-Stokes problem:

$$
\begin{aligned}
-\nu \Delta \mathbf{u}+(\mathbf{u} \cdot \nabla) \mathbf{u}+\nabla p & =\mathbf{f} \text { in } \Omega, \\
\operatorname{div} \mathbf{u} & =\mathbf{0} \text { in } \Omega, \\
\mathbf{u} & =\mathbf{0} \text { on } \Gamma, \\
\int_{\Omega} p & =0,
\end{aligned}
$$

where $\Omega \subset \mathbb{R}^{n}, n \in\{2,3\}$, is a polyhedral domain, $\nu>0$ is the fluid viscosity, $\mathbf{u}$ is the velocity, $p$ is the pressure, and $\mathbf{f}$ is a source term. In particular, we are interested in pseudostress-type mixed variational formulations to solve problem (1.1).

Pseudostress-based formulations, applied to the Stokes problem, have been extensively studied during the last decades (see e.g. [5], [14], [20], [22], and the references therein). This study has been extended to solve important problems in engineering, such as the Stokes-Darcy coupled problem and transport problems (see, for instance, [1], [21], [24], [25]).

There are two main advantages to utilize this kind of formulations. On the one hand, pseudostress-based formulations have a natural applicability to non-Newtonian flows. Indeed, since in this case the constitutive equation is nonlinear, the stress can not be eliminated, and hence it becomes an unavoidable unknown in the corresponding solvability analysis. Actually, the main advantage of this kind of formulations is that their allows for a unified analysis for linear and nonlinear flows (see [11], [19], [23], [28]). On the other hand, when using pseudostressbased formulations, further variables of interest can be computed as a simple postprocess of the pseudostress, and then, they can be approximated without loosing any accuracy. In fact, after computing the pseudostress tensor $\boldsymbol{\sigma}:=\nu \nabla \mathbf{u}-p \mathrm{I}$ one can easily obtain the fluid stress tensor $\tilde{\boldsymbol{\sigma}}:=\nu\left(\nabla \mathbf{u}+\nabla \mathbf{u}^{t}\right)-p \mathrm{I}$, the fluid pressure $p$, the fluid vorticity $\boldsymbol{\omega}:=\frac{1}{2}\left(\nabla \mathbf{u}-(\nabla \mathbf{u})^{t}\right)$, and the fluid velocity gradient $\nabla \mathbf{u}$, respectively, as follows:

$$
\tilde{\boldsymbol{\sigma}}=\boldsymbol{\sigma}-\frac{1}{n} \operatorname{tr}(\boldsymbol{\sigma}) \mathrm{I}+\boldsymbol{\sigma}^{t}, \quad p=-\frac{1}{n} \operatorname{tr}(\boldsymbol{\sigma}), \quad \boldsymbol{\omega}=\frac{1}{2 \nu}\left(\boldsymbol{\sigma}-\boldsymbol{\sigma}^{t}\right) \quad \text { and } \quad \nabla \mathbf{u}=\frac{1}{\nu}\left(\boldsymbol{\sigma}-\frac{1}{n} \operatorname{tr}(\boldsymbol{\sigma}) \mathrm{I}\right) .
$$

Now, concerning mixed formulations for the Navier-Stokes equations, we first mention the works of Farhloul et al. (see [12] and [13]), where the authors extend the analysis of dual-mixed formulations for the Stokes equations to the Navier-Stokes problem. They propose quasi-optimal convergent numerical methods for the fluid flow problem considering the strain tensor (in [12]) and the velocity gradient tensor (in [13]) as the main unknowns of the corresponding systems. In [8] (see also [6] and [7]), Cai et al. have extended the analysis of pseudostress-based mixed methods for the Stokes problem to the Navier-Stokes equations. They introduce and analyze a conforming $\mathbf{H}$ (div) method for a pseudostress-based mixed formulation which turns to be of accuracy $O\left(h^{k+1-n / 6}\right)(n=2,3)$ in the $L^{3}$ norm. Finally, in [29], Howell and Walkington, present a dual-mixed finite element method for the Navier-Stokes problem, where the main unknowns are the velocity-gradient (in $L^{2}$ ), the velocity (in $L^{2}$ ) and a modified version of the pseudostress (or stress) tensor (in $\mathbf{H}$ (div)) that links the pseudostress tensor and the convective term. The authors prove well-posedness of the continuous formulation utilizing the classical Babuška-Brezzi theory, the fact that the velocity can be seen as a function in $H^{1}$ (Lemma 2.4), and a fixed-point argument. The first tool involves inf-sup conditions which are not easy to prove for the classical 
$\mathbf{H}$ (div )-conforming discrete spaces. In order to deal with this issue, the authors introduce new finite element subspaces for the corresponding unknowns, which turn to be expensive.

In this work we introduce a new augmented mixed finite element method for problem (1.1). Similarly to [29], we introduce a "nonlinear-pseudostress" tensor (in $\mathbf{H}(\operatorname{div})$ ), linking the pseudostress tensor and the convective term, which together to the velocity (in $H^{1}$ ), are the main unknowns of the system. The pressure is eliminated by using the incompressibility property, and can be recovered as a simple postprocess of the nonlinear-pseudostress tensor. Since the velocity is kept as a function in $H^{1}$, we utilize similar techniques developed in [16] and [17] for the linear elasticity problem (see also [14] for similar results applied to the Stokes problem), and augment the mixed formulation with Galerkin least-squares type terms arising from the constitutive and equilibrium equations of the Navier-Stokes equations and from the Dirichlet boundary condition, which are multiplied by stabilization parameters that are chosen in such a way that the resulting continuous formulation becomes well-posed. The introduction of these terms allows us to circumvent the necessity of proving inf-sup conditions, and as a result, to relax the hypotheses on the corresponding discrete subspaces. In this way, the classical Banach's fixed point Theorem and Lax-Milgram's Lemma can be applied to prove well-posedness of the continuous problem. Analogously, and considering any pair of conforming subspaces for the nonlinear-pseudostress tensor and the fluid velocity, we establish well-posedness and the corresponding Cea's estimate of the associated Galerkin scheme. In particular, making use of Raviart-Thomas elements of degree $k$ for the nonlinear-pseudostress tensor, and continuous piecewise polynomial elements of degree $k+1$ for the velocity, we obtain optimal rate of convergence, which is one of the principal advantages of our method.

The rest of this paper is organized as follows. In Section 2 we present the main aspects of the continuous problem. We reformulate the fluid flow problem as an equivalent first-order set of equations. Then, we multiply the corresponding equations by suitable test functions and integrate by parts to derive our augmented mixed variational formulation. In Section 3 we choose appropriate stabilization parameters, and apply the classical Banach's fixed point Theorem and Lax-Milgram's Lemma to prove unique solvability and stability of the continuous formulation. In Section 4 we introduce and analyze the associated Galerkin scheme. We proceed analogously to the continuous case, and establish well-posedness of the corresponding discrete problem, considering any subspace for the nonlinear-pseudostress tensor and the velocity. In

addition, we establish the corresponding Cea's estimate and provide a suitable choice of finite element spaces ensuring optimal rate of convergence. In Section 5 we present two iterative strategies to compute the solutions of the aforementioned augmented scheme, and analyze their convergence. Finally, several numerical results illustrating the good performance of the method, are reported in Section 6.

\section{Continuous problem}

\subsection{Preliminaries}

Let us first introduce some general functional spaces. If $\mathcal{O}$ is a domain and $r \in \mathbb{R}$, we define

$$
\mathbf{H}^{r}(\mathcal{O}):=\left[\mathrm{H}^{r}(\mathcal{O})\right]^{n}, \quad \mathbb{H}^{r}(\mathcal{O}):=\left[\mathrm{H}^{r}(\mathcal{O})\right]^{n \times n} .
$$

In the particular case $r=0$ we usually write $\mathbf{L}^{2}(\mathcal{O})$, and $\mathbb{L}^{2}(\mathcal{O})$, instead of $\mathbf{H}^{0}(\mathcal{O})$, and $\mathbb{H}^{0}(\mathcal{O})$, respectively. The corresponding norms are denoted by $\|\cdot\|_{r, \mathcal{O}}\left(\right.$ for $\mathrm{H}^{r}(\mathcal{O}), \mathbf{H}^{r}(\mathcal{O})$, and $\mathbb{H}^{r}(\mathcal{O})$ ). 
Also, the Hilbert space

$$
\mathbf{H}(\operatorname{div} ; \mathcal{O}):=\left\{\mathbf{w} \in \mathbf{L}^{2}(\mathcal{O}): \quad \operatorname{div} \mathbf{w} \in L^{2}(\mathcal{O})\right\}
$$

is standard in the realm of mixed problems (see [4], [18] [15] for instance), where div is the usual divergence operator acting on vector fields. The space of matrix valued functions whose rows belong to $\mathbf{H}(\operatorname{div} ; \mathcal{O})$ will be denoted $\mathbb{H}(\operatorname{div} ; \mathcal{O})$. The Hilbert norms of $\mathbf{H}(\operatorname{div} ; \mathcal{O})$ and $\mathbb{H}(\mathbf{d i v} ; \mathcal{O})$ are denoted by $\|\cdot\|_{\operatorname{div} ; \mathcal{O}}$ and $\|\cdot\|_{\operatorname{div} ; \mathcal{O}}$, respectively. Note that if $\mathbf{S} \in \mathbb{H}(\mathbf{d i v} ; \mathcal{O})$, then $\operatorname{div} \mathbf{S} \in \mathbf{L}^{2}(\mathcal{O})$. Note also that $\mathbb{H}(\mathbf{d i v} ; \mathcal{O})$ can be characterized as the space of matrix valued functions $\mathbf{S}$ such that $\mathbf{c}^{t} \mathbf{S} \in \mathbf{H}(\operatorname{div} ; \mathcal{O})$ for any constant column vector $\mathbf{c}$. In addition, we have the following decomposition:

$$
\mathbb{H}(\operatorname{div} ; \mathcal{O})=\mathbb{H}_{0}(\operatorname{div} ; \mathcal{O}) \oplus P_{0}(\mathcal{O}) \mathrm{I}
$$

where

$$
\mathbb{H}_{0}(\operatorname{div} ; \mathcal{O}):=\left\{\mathbf{S} \in \mathbb{H}(\operatorname{div} ; \mathcal{O}): \quad \int_{\mathcal{O}} \operatorname{tr} \mathbf{S}=0\right\}
$$

I is the $n \times n$ identity matrix, $P_{0}(\mathcal{O})$ is the space of constant polynomials on $\mathcal{O}$, and $\operatorname{tr}$ stands for the usual trace of tensor. More precisely, each $\mathbf{S} \in \mathbb{H}(\mathbf{d i v} ; \mathcal{O})$ can be decomposed uniquely as:

$$
\mathbf{S}=\mathbf{S}_{0}+c \mathrm{I}, \quad \text { with } \quad \mathbf{S}_{0} \in \mathbb{H}_{0}(\operatorname{div} ; \mathcal{O}) \quad \text { and } \quad c:=\frac{1}{n|\mathcal{O}|} \int_{\mathcal{O}} \operatorname{tr} \mathbf{S} \in \mathbb{R} .
$$

Finally, for the sake of simplicity, in what follows we denote

$$
(u, v)_{\Omega}:=\int_{\Omega} u v, \quad(\mathbf{u}, \mathbf{v})_{\Omega}:=\int_{\Omega} \mathbf{u} \cdot \mathbf{v}, \quad(\mathbf{u}, \mathbf{v})_{\Gamma}:=\int_{\Gamma} \mathbf{u} \cdot \mathbf{v} \quad \text { and } \quad(\mathbf{T}, \mathbf{S})_{\Omega}:=\int_{\Omega} \mathbf{T}: \mathbf{S} .
$$

\subsection{The first-order set of equations}

As we already mentioned, in order to describe our mixed variational formulation, we first rewrite problem (1.1) as an equivalent first-order set of equations. We begin by introducing the "nonlinear-pseudostress" tensor

$$
\mathbf{T}:=\nu \nabla \mathbf{u}-p \mathbf{I}-\mathbf{u} \otimes \mathbf{u} \quad \text { in } \quad \Omega .
$$

From the incompressibility condition $\operatorname{div} \mathbf{u}=\operatorname{tr}(\nabla \mathbf{u})=0$ in $\Omega$, it is not difficult to see that

$$
\operatorname{div}(\mathbf{u} \otimes \mathbf{u})=(\mathbf{u} \cdot \nabla) \mathbf{u} \quad \text { in } \quad \Omega \quad \text { and } \quad \operatorname{tr}(\mathbf{T})=-n p-\operatorname{tr}(\mathbf{u} \otimes \mathbf{u}) \quad \text { in } \quad \Omega .
$$

In particular, the second equation in (2.5) allows us to write the pressure $p$ in terms of the tensor $\mathbf{T}$ and the velocity $\mathbf{u}$ as

$$
p=-\frac{1}{n}(\operatorname{tr}(\mathbf{T})+\operatorname{tr}(\mathbf{u} \otimes \mathbf{u})) \quad \text { in } \quad \Omega,
$$

which in turns, together with (2.4), leads us to the equation

$$
\mathbf{T}^{d}=\nu \nabla \mathbf{u}-(\mathbf{u} \otimes \mathbf{u})^{d} \quad \text { in } \quad \Omega,
$$

where

$$
\mathbf{S}^{d}:=\mathbf{S}-\frac{1}{n}(\operatorname{tr} \mathbf{S}) \mathrm{I},
$$


is the deviatoric part of tensor $\mathbf{S}$.

On the other hand, from (2.4), and the first equations of (1.1) and (2.5), we easily get the equilibrium equation

$$
-\operatorname{div} \mathbf{T}=\mathbf{f} \quad \text { in } \Omega .
$$

Finally, from (2.6) we observe that the condition $\int_{\Omega} p=0$, ensuring the uniqueness of solution of problem (1.1), is equivalent to

$$
(\operatorname{tr}(\mathbf{T})+\operatorname{tr}(\mathbf{u} \otimes \mathbf{u}), 1)_{\Omega}=0 .
$$

According to the above, we rewrite equations (1.1) equivalently as follows

$$
\begin{aligned}
& \mathbf{T}^{d}=\nu \nabla \mathbf{u}-(\mathbf{u} \otimes \mathbf{u})^{d} \quad \text { in } \quad \Omega, \quad-\operatorname{div} \mathbf{T}=\mathbf{f} \quad \text { in } \quad \Omega, \\
& \mathbf{u}=\mathbf{0} \quad \text { on } \quad \Gamma, \quad(\operatorname{tr}(\mathbf{T}), 1)_{\Omega}=-(\operatorname{tr}(\mathbf{u} \otimes \mathbf{u}), 1)_{\Omega} .
\end{aligned}
$$

where the unknowns of the system are the tensor $\mathbf{T}$ and the velocity $\mathbf{u}$. The pressure $p$ can be easily computed as a postprocess of the solution by using (2.6). Here,

$$
\nabla \mathbf{u}=\left(\frac{\partial u_{i}}{\partial x_{j}}\right)_{1 \leq i, j \leq n}, \quad \text { and } \quad \operatorname{div} \mathbf{T}=\left(\operatorname{div}\left(t_{i 1}, \ldots, t_{i n}\right)\right)_{1 \leq i \leq n} .
$$

Remark 2.1 Notice that, utilizing the solution of (2.10), besides recovering the pressure p, we can also compute other variables of interest, such as the stress tensor $\tilde{\boldsymbol{\sigma}}=\nu\left(\nabla \mathbf{u}+(\nabla \mathbf{u})^{t}\right)-p \mathrm{I}$, the velocity gradient $\nabla \mathbf{u}$ and the vorticity $\boldsymbol{\omega}:=\frac{1}{2}\left(\nabla \mathbf{u}-(\nabla \mathbf{u})^{t}\right)$, respectively, as follows

$$
\begin{aligned}
\tilde{\boldsymbol{\sigma}} & =\mathbf{T}^{d}+(\mathbf{u} \otimes \mathbf{u})^{d}+\mathbf{T}^{t}+\mathbf{u} \otimes \mathbf{u} \\
\nabla \mathbf{u} & =\frac{1}{\nu}\left(\mathbf{T}^{d}+(\mathbf{u} \otimes \mathbf{u})^{d}\right) \\
\boldsymbol{\omega} & =\frac{1}{2 \nu}\left(\mathbf{T}-\mathbf{T}^{t}\right) .
\end{aligned}
$$

These formulae will be employed later to approximate this variables as a simple postprocess of the finite element solutions, conserving the same rate of convergence.

\subsection{The augmented mixed variational problem}

We now turn to the derivation of the weak formulation of (2.10). To do this we first test the first equation of $(2.10)$ with arbitrary $\mathbf{S} \in \mathbb{H}(\mathbf{d i v} ; \Omega)$, integrate by parts, utilize the Dirichlet boundary condition $\mathbf{u}=\mathbf{0}$ on $\Gamma$, and the identity $\mathbf{T}^{d}: \mathbf{S}=\mathbf{T}^{d}: \mathbf{S}^{d}$, to obtain

$$
\left(\mathbf{T}^{d}, \mathbf{S}^{d}\right)_{\Omega}+\nu(\operatorname{div} \mathbf{S}, \mathbf{u})_{\Omega}+\left(\mathbf{u} \otimes \mathbf{u}, \mathbf{S}^{d}\right)_{\Omega}=0
$$

In addition, the equilibrium equation $\operatorname{div} \mathbf{T}=-\mathbf{f}$ is imposed weakly as follows

$$
\nu(\operatorname{div} \mathbf{T}, \mathbf{v})_{\Omega}=-\nu(\mathbf{f}, \mathbf{v})_{\Omega},
$$

where $\mathbf{v}$ is a suitable test function. Observe that the term $\left(\mathbf{u} \otimes \mathbf{u}, \mathbf{S}^{d}\right)_{\Omega}$ in $(2.12)$ requires the velocity $\mathbf{u}$ to be in a space smaller than $\mathbf{L}^{2}(\Omega)$. Then, as we will see later, in order to obtain an optimal convergent discrete scheme, we propose to consider the velocity $\mathbf{u}$ in $\mathbf{H}^{1}(\Omega)$. 
Consequently, in order to ensure well-posedness of the resulting variational formulation, we propose to enrich our formulation with the following residual terms arising from the constitutive, equilibrium equations and the Dirichlet boundary condition

$$
\begin{gathered}
\kappa_{1}(\operatorname{div} \mathbf{T}, \operatorname{div} \mathbf{S})_{\Omega}=-\kappa_{1}(\mathbf{f}, \operatorname{div} \mathbf{S})_{\Omega} \\
\kappa_{2}\left(\nu \nabla \mathbf{u}-\mathbf{T}^{d}-(\mathbf{u} \otimes \mathbf{u})^{d}, \nabla \mathbf{v}\right)_{\Omega}=0 \\
\kappa_{3}(\mathbf{u}, \mathbf{v})_{\Gamma}=0
\end{gathered}
$$

for all $\mathbf{S} \in \mathbb{H}(\operatorname{div} ; \Omega)$ and $\mathbf{v} \in \mathbf{H}^{1}(\Omega)$, where $\kappa_{1}, \kappa_{2}$ and $\kappa_{3}$ are positive parameters to be specified later.

Then, from (2.12)-(2.16) we obtain the variational problem: Find $(\mathbf{T}, \mathbf{u}) \in \mathbb{H}(\mathbf{d i v} ; \Omega) \times$ $\mathbf{H}^{1}(\Omega)$, such that $(\operatorname{tr}(\mathbf{T})+\operatorname{tr}(\mathbf{u} \otimes \mathbf{u}), 1)_{\Omega}=0$, and

$$
\begin{aligned}
&\left(\mathbf{T}^{d}, \mathbf{S}^{d}\right)_{\Omega}+\kappa_{1}(\operatorname{div} \mathbf{T}, \operatorname{div} \mathbf{S})_{\Omega}+\nu(\operatorname{div} \mathbf{S}, \mathbf{u})_{\Omega}+\left(\mathbf{u} \otimes \mathbf{u}, \mathbf{S}^{d}\right)_{\Omega}=-\kappa_{1}(\mathbf{f}, \operatorname{div} \mathbf{S})_{\Omega}, \\
&-\nu(\operatorname{div} \mathbf{T}, \mathbf{v})_{\Omega}+\kappa_{2}\left(\nu \nabla \mathbf{u}-\mathbf{T}^{d}-(\mathbf{u} \otimes \mathbf{u})^{d}, \nabla \mathbf{v}\right)_{\Omega}+\kappa_{3}(\mathbf{u}, \mathbf{v})_{\Gamma}=\nu(\mathbf{f}, \mathbf{v})_{\Omega},
\end{aligned}
$$

for all $(\mathbf{S}, \mathbf{v}) \in \mathbb{H}(\mathbf{d i v} ; \Omega) \times \in \mathbf{H}^{1}(\Omega)$.

Let us now define the tensor

$$
\mathbf{T}_{0}:=\mathbf{T}+\frac{1}{n|\Omega|}(\operatorname{tr}(\mathbf{u} \otimes \mathbf{u}), 1)_{\Omega} \mathrm{I}
$$

It is clear that

$$
\mathbf{T}_{0} \in \mathbb{H}_{0}(\mathbf{d i v} ; \Omega) \quad \text { if and only if } \quad(\operatorname{tr}(\mathbf{T})+\operatorname{tr}(\mathbf{u} \otimes \mathbf{u}), 1)_{\Omega}=0 .
$$

In this way, thanks to (2.18) and (2.3), problem (2.17) can be reformulated equivalently as: Find $\left(\mathbf{T}_{0}, \mathbf{u}\right) \in \mathbb{H}_{0}(\operatorname{div} ; \Omega) \times \mathbf{H}^{1}(\Omega)$, such that

$$
\begin{aligned}
&\left(\mathbf{T}_{0}^{d}, \mathbf{S}^{d}\right)_{\Omega}+\kappa_{1}\left(\operatorname{div} \mathbf{T}_{0}, \operatorname{div} \mathbf{S}\right)_{\Omega}+\nu(\operatorname{div} \mathbf{S}, \mathbf{u})_{\Omega}+\left(\mathbf{u} \otimes \mathbf{u}, \mathbf{S}^{d}\right)_{\Omega}=-\kappa_{1}(\mathbf{f}, \operatorname{div} \mathbf{S})_{\Omega} \\
&-\nu\left(\operatorname{div} \mathbf{T}_{0}, \mathbf{v}\right)_{\Omega}+\kappa_{2}\left(\nu \nabla \mathbf{u}-\mathbf{T}_{0}^{d}-(\mathbf{u} \otimes \mathbf{u})^{d}, \nabla \mathbf{v}\right)_{\Omega}+\kappa_{3}(\mathbf{u}, \mathbf{v})_{\Gamma}=\nu(\mathbf{f}, \mathbf{v})_{\Omega}
\end{aligned}
$$

for all $(\mathbf{S}, \mathbf{v}) \in \mathbb{H}_{0}(\mathbf{d i v} ; \Omega) \times \in \mathbf{H}^{1}(\Omega)$.

The following lemma establishes that problems (2.17) and (2.19) are equivalent. Its proof is straightforward.

Lemma 2.2 If $(\mathbf{T}, \mathbf{u})$ is a solution of $(2.17)$, then $\left(\mathbf{T}_{0}, \mathbf{u}\right)=\left(\mathbf{T}+\frac{1}{n|\Omega|}(\operatorname{tr}(\mathbf{u} \otimes \mathbf{u}), 1)_{\Omega} \mathrm{I}, \mathbf{u}\right)$ is a solution of (2.19). Conversely, if $\left(\mathbf{T}_{0}, \mathbf{u}\right)$ is a solution of $(2.19)$, then $(\mathbf{T}, \mathbf{u})=\left(\mathbf{T}_{0}-\frac{1}{n|\Omega|}(\operatorname{tr}(\mathbf{u} \otimes\right.$ $\left.\mathbf{u}), 1)_{\Omega} \mathrm{I}, \mathbf{u}\right)$ is a solution of $(2.17)$.

As a consequence of the above, in what follows we focus on analyzing problem (2.19).

\section{Analysis of the continuous problem}

In this section we analyze the well-posedness of problem (2.19). To that end, we first rewrite our problem in a variational setting and state the main properties of the forms involved. 


\subsection{Variational setting}

First, let us define the global unknown and space:

$$
\underline{\phi}:=\left(\mathbf{T}_{0}, \mathbf{u}\right) \in \mathbf{X}:=\mathbb{H}_{0}(\operatorname{div} ; \Omega) \times \mathbf{H}^{1}(\Omega),
$$

where $\mathbf{X}$ is endowed with the norm

$$
\|\underline{\boldsymbol{\psi}}\|_{\mathbf{X}}^{2}:=\|\mathbf{S}\|_{\mathbf{d i v}, \Omega}^{2}+\|\mathbf{v}\|_{1, \Omega}^{2} \quad \forall \underline{\boldsymbol{\psi}}=(\mathbf{S}, \mathbf{v}) \in \mathbf{X} .
$$

Then, defining the forms

$$
\begin{aligned}
\mathbf{A}(\underline{\phi}, \underline{\boldsymbol{\psi}}):= & \left(\mathbf{T}^{d}, \mathbf{S}^{d}\right)_{\Omega}+\kappa_{1}(\operatorname{div} \mathbf{T}, \operatorname{div} \mathbf{S})_{\Omega}+\kappa_{2} \nu(\nabla \mathbf{u}, \nabla \mathbf{v})_{\Omega}+\kappa_{3}(\mathbf{u}, \mathbf{v})_{\Gamma} \\
& -\nu(\operatorname{div} \mathbf{T}, \mathbf{v})_{\Omega}+\nu(\operatorname{div} \mathbf{S}, \mathbf{u})_{\Omega}-\kappa_{2}\left(\mathbf{T}^{d}, \nabla \mathbf{v}\right)_{\Omega}, \\
\mathbf{C}(\underline{\boldsymbol{\zeta}} ; \underline{\phi}, \underline{\boldsymbol{\psi}}):= & \left(\mathbf{u} \otimes \mathbf{z}, \mathbf{S}^{d}\right)_{\Omega}-\kappa_{2}\left((\mathbf{u} \otimes \mathbf{z})^{d}, \nabla \mathbf{v}\right)_{\Omega},
\end{aligned}
$$

for all $\underline{\phi}=(\mathbf{T}, \mathbf{u}), \underline{\boldsymbol{\psi}}=(\mathbf{S}, \mathbf{v}), \underline{\boldsymbol{\zeta}}=(\mathbf{R}, \mathbf{z})$ in $\mathbf{X}$, and the functional

$$
\mathbf{F}(\underline{\boldsymbol{\psi}}):=-\kappa_{1}(\mathbf{f}, \operatorname{div} \mathbf{S})_{\Omega}+\nu(\mathbf{f}, \mathbf{v})_{\Omega} \quad \forall \underline{\boldsymbol{\psi}}=(\mathbf{S}, \mathbf{v}) \in \mathbf{X},
$$

the variational problem (2.19) reads: Find $\underline{\phi} \in \mathbf{X}$, such that

$$
\mathbf{A}(\underline{\phi}, \underline{\psi})+\mathbf{C}(\underline{\phi} ; \underline{\phi}, \underline{\psi})=\mathbf{F}(\underline{\psi}) \quad \forall \underline{\psi}=(\mathbf{S}, \mathbf{v}) \in \mathbf{X} .
$$

We now turn to discuss the stability properties of the forms involved. To do this we first establish the following well known inequalities. For (3.4) we refer to Lemma 3.1 in [2] or Chapter IV in [4], whereas for inequality (3.5) we refer to [9] and Chapter I in [15].

$$
\begin{gathered}
C_{d}\|\mathbf{S}\|_{0, \Omega}^{2} \leq\left\|\mathbf{S}^{d}\right\|_{0, \Omega}^{2}+\|\operatorname{div} \mathbf{S}\|_{0, \Omega}^{2} \quad \forall \mathbf{S} \in \mathbb{H}_{0}(\operatorname{div} ; \Omega), \\
C_{p}\|v\|_{1, \Omega}^{2} \leq|v|_{1, \Omega}^{2}+\|v\|_{0, \Gamma}^{2} \leq \tilde{C}_{p}\|v\|_{1, \Omega}^{2} \quad \forall v \in \mathrm{H}^{1}(\Omega),
\end{gathered}
$$

with $C_{d}, C_{p}$ and $\tilde{C}_{p}$, only depending on $\Omega$. Notice that the inequality on left hand side of (3.5) is nothing but the well known generalized Poincaré inequality.

Now, for the bilinear form A, we observe that, thanks to (3.5) and the Hölder's inequality, for any positive constants $\kappa_{1}, \kappa_{2}$, and $\kappa_{3}$, there holds

$$
|\mathbf{A}(\underline{\phi}, \underline{\psi})| \leq C_{\mathbf{A}}\|\underline{\phi}\|_{\mathbf{X}}\|\underline{\psi}\|_{\mathbf{X}}, \quad \forall \underline{\phi}, \underline{\psi} \in \mathbf{X},
$$

with $C_{\mathbf{A}}>0$ depending only on $\nu, \kappa_{1}, \kappa_{2}, \kappa_{3}$ and $\tilde{C}_{p}$.

Now, for the form $\mathbf{C}$, recalling that $H^{1}$ is continuously embedded in $L^{4}$, from Hölder's inequality, we obtain

$$
\begin{aligned}
|\mathbf{C}(\underline{\boldsymbol{\zeta}} ; \underline{\boldsymbol{\phi}}, \underline{\boldsymbol{\psi}})| & \leq\left(1+\kappa_{2}^{2}\right)^{1 / 2}\|\mathbf{z}\|_{L^{4}(\Omega)}\|\mathbf{u}\|_{L^{4}(\Omega)}\|\underline{\boldsymbol{\psi}}\|_{\mathbf{X}} \\
& \leq\left(1+\kappa_{2}^{2}\right)^{1 / 2} C_{\text {sob }}^{2}\|\mathbf{z}\|_{1, \Omega}\|\mathbf{u}\|_{1, \Omega}\|\underline{\boldsymbol{\psi}}\|_{\mathbf{X}} \\
& \leq C_{\mathbf{C}}\|\underline{\boldsymbol{\zeta}}\|_{\mathbf{X}}\|\underline{\boldsymbol{\phi}}\|_{\mathbf{X}}\|\underline{\boldsymbol{\psi}}\|_{\mathbf{X}},
\end{aligned}
$$

for all $\underline{\boldsymbol{\zeta}}=(\mathbf{R}, \mathbf{z}), \underline{\boldsymbol{\phi}}=(\mathbf{T}, \mathbf{u})$, and $\underline{\boldsymbol{\psi}} \in \mathbf{X}$, with $C_{\mathbf{C}}:=C_{\text {sob }}^{2}\left(1+\kappa_{2}^{2}\right)^{1 / 2}$. Here, $C_{\text {sob }}>0$ is the constant of the corresponding Sobolev inequality.

We now turn to prove the ellipticity of $\mathbf{A}$ on $\mathbf{X}$. This result is established next. 
Lemma 3.1 Assume that $\kappa_{1}, \kappa_{3}>0$ and $0<\kappa_{2}<2 \nu$. Then there exists $\alpha_{\mathbf{A}}>0$, such that

$$
\mathbf{A}(\underline{\boldsymbol{\psi}}, \underline{\boldsymbol{\psi}}) \geq \alpha_{\mathbf{A}}\|\underline{\boldsymbol{\psi}}\|_{\mathbf{X}}^{2} \quad \forall \underline{\boldsymbol{\psi}} \in \mathbf{X} .
$$

Proof. Given $\underline{\psi}:=(\mathbf{S}, \mathbf{v}) \in \mathbf{X}$, from the definition of A, inequalities (3.4), (3.5), and Hölder's inequality, we have

$$
\begin{aligned}
\mathbf{A}(\underline{\boldsymbol{\psi}}, \underline{\boldsymbol{\psi}}) & =\left\|\mathbf{S}^{d}\right\|_{0, \Omega}^{2}+\kappa_{1}\|\operatorname{div} \mathbf{S}\|_{0, \Omega}^{2}+\kappa_{2} \nu|\mathbf{v}|_{1, \Omega}^{2}+\kappa_{3}\|\mathbf{v}\|_{0, \Gamma}^{2}-\kappa_{2}\left(\mathbf{S}^{d}, \nabla \mathbf{v}\right)_{\Omega} \\
& \geq\left\|\mathbf{S}^{d}\right\|_{0, \Omega}^{2}+\kappa_{1}\|\operatorname{div} \mathbf{S}\|_{0, \Omega}^{2}+\kappa_{2} \nu|\mathbf{v}|_{1, \Omega}^{2}+\kappa_{3}\|\mathbf{v}\|_{0, \Gamma}^{2}-\frac{1}{2}\left\|\mathbf{S}^{d}\right\|_{0, \Omega}^{2}-\frac{\kappa_{2}^{2}}{2}|\mathbf{v}|_{1, \Omega}^{2} \\
& \geq \frac{1}{2}\left\|\mathbf{S}^{d}\right\|_{0, \Omega}^{2}+\kappa_{1}\|\operatorname{div} \mathbf{S}\|_{0, \Omega}^{2}+\frac{\kappa_{2}}{2}\left(2 \nu-\kappa_{2}\right)|\mathbf{v}|_{1, \Omega}^{2}+\kappa_{3}\|\mathbf{v}\|_{0, \Gamma}^{2} \\
& \geq \frac{1}{2} \min \left\{1, \kappa_{1}\right\} C_{d}\|\mathbf{S}\|_{0, \Omega}^{2}+\frac{\kappa_{1}}{2}\|\operatorname{div} \mathbf{S}\|_{0, \Omega}^{2}+\frac{1}{2} \min \left\{\kappa_{2}\left(2 \nu-\kappa_{2}\right), 2 \kappa_{3}\right\} C_{p}\|\mathbf{v}\|_{1, \Omega}^{2} .
\end{aligned}
$$

Then, the result follows with $\alpha_{\mathbf{A}}=\frac{1}{2} \min \left\{\min \left\{1, \kappa_{1}\right\} C_{d}, \kappa_{1}, \min \left\{\kappa_{2}\left(2 \nu-\kappa_{2}\right), 2 \kappa_{3}\right\} C_{p}\right\}$.

Remark 3.2 Observe that if $\kappa_{2} \in(0,2 \nu), \kappa_{1}=\kappa_{2}\left(2 \nu-\kappa_{2}\right)$ and $\kappa_{3}=\frac{1}{2} \kappa_{2}\left(2 \nu-\kappa_{2}\right)$, then for any viscosity constant $\nu \in(0,1]$, the ellipticity constant $\alpha_{\mathbf{A}}$ becomes

$$
\alpha_{\mathbf{A}}=\frac{1}{2} \kappa_{2}\left(2 \nu-\kappa_{2}\right) \min \left\{C_{d}, 1, C_{p}\right\}
$$

which reaches its maximum value when $\kappa_{2}=\nu$.

We end this section by establishing the boundedness of the functional $\mathbf{F}$ :

$$
\begin{aligned}
|\mathbf{F}(\underline{\boldsymbol{\psi}})| & \leq\|\mathbf{f}\|_{0, \Omega}\left(\kappa_{1}\|\operatorname{div} \mathbf{S}\|_{0, \Omega}+\nu\|\mathbf{v}\|_{0, \Omega}\right) \\
& \leq\|\mathbf{f}\|_{0, \Omega}\left(\kappa_{1}^{2}+\nu^{2}\right)^{1 / 2}\|\underline{\boldsymbol{\psi}}\|_{\mathbf{X}},
\end{aligned}
$$

for all $\underline{\psi}=(\mathbf{S}, \mathbf{v}) \in \mathbf{X}$.

\subsection{The main result}

Now, we are in position of providing the main result of this section, namely, existence, uniqueness and stability of problem (3.3). To that end we first introduce the bounded set

$$
\mathbf{K}:=\left\{\underline{\boldsymbol{\psi}} \in \mathbf{X}:\|\underline{\boldsymbol{\psi}}\|_{\mathbf{X}} \leq \frac{2\left(\kappa_{1}^{2}+\nu^{2}\right)^{1 / 2}}{\alpha_{\mathbf{A}}}\|\mathbf{f}\|_{0, \Omega}\right\},
$$

and the mapping

$$
\mathcal{J}: \mathbf{K} \rightarrow \mathbf{K}, \quad \underline{\boldsymbol{\zeta}} \rightarrow \mathcal{J}(\underline{\boldsymbol{\zeta}})=\underline{\phi}
$$

with $\underline{\zeta}$ and $\underline{\phi}$ satisfying

$$
\mathbf{A}(\underline{\phi}, \underline{\psi})+\mathbf{C}(\underline{\boldsymbol{\zeta}} ; \underline{\phi}, \underline{\psi})=\mathbf{F}(\underline{\psi}) \quad \forall \underline{\psi} \in \mathbf{X} .
$$

Let us observe that $\underline{\phi} \in \mathbf{X}$ is a solution to (3.3), if and only if, $\mathcal{J}(\underline{\phi})=\underline{\phi}$. In this way, to prove well-posedness of problem (3.3), in what follows we equivalently prove that $\mathcal{J}$ has a unique fixed point by means of the classical Banach's fixed point theorem. Before doing this, we now provide the following lemma establishing the well-definedness of operator $\mathcal{J}$. 
Lemma 3.3 Assume that

$$
\frac{4 C_{\mathbf{C}}\left(\kappa_{1}^{2}+\nu^{2}\right)^{1 / 2}}{\alpha_{\mathbf{A}}^{2}}\|\mathbf{f}\|_{0, \Omega} \leq 1,
$$

with $C_{\mathbf{C}}$ and $\alpha_{\mathbf{A}}$ being the constants in (3.7) and (3.8), respectively. Assume further that $\kappa_{1}, \kappa_{3}>0$ and $0<\kappa_{2}<2 \nu$. Then, given $\underline{\boldsymbol{\zeta}} \in \mathbf{K}$, there exists a unique $\underline{\boldsymbol{\phi}} \in \mathbf{K}$ such that $\mathcal{J}(\underline{\zeta})=\underline{\phi}$.

Proof. Let $\underline{\boldsymbol{\zeta}} \in \mathbf{K}$. From (3.7) and (3.8), there holds

$$
\begin{aligned}
\mathbf{A}(\underline{\boldsymbol{\psi}}, \underline{\boldsymbol{\psi}})+\mathbf{C}(\underline{\boldsymbol{\zeta}} ; \underline{\boldsymbol{\psi}}, \underline{\boldsymbol{\psi}}) \geq \mathbf{A}(\underline{\boldsymbol{\psi}}, \underline{\boldsymbol{\psi}})-|\mathbf{C}(\underline{\boldsymbol{\zeta}} ; \underline{\boldsymbol{\psi}}, \underline{\boldsymbol{\psi}})| & \forall \underline{\boldsymbol{\psi}} \in \mathbf{X}, \\
& \geq\left(\alpha_{\mathbf{A}}-C_{\mathbf{C}}\|\underline{\boldsymbol{\zeta}}\|_{\mathbf{X}}\right)\|\underline{\boldsymbol{\psi}}\|_{\mathbf{X}}^{2} \quad \forall \underline{\boldsymbol{\psi}} \in \mathbf{X} .
\end{aligned}
$$

In turn, from the definition of set $\mathbf{K}$, and assumption (3.13), we easily get

$$
C_{\mathbf{C}}\|\underline{\boldsymbol{\zeta}}\|_{\mathbf{X}} \leq \frac{2 C_{\mathbf{C}}\left(\kappa_{1}^{2}+\nu^{2}\right)^{1 / 2}}{\alpha_{\mathbf{A}}}\|\mathbf{f}\|_{0, \Omega} \leq \frac{\alpha_{\mathbf{A}}}{2} .
$$

Hence, combining (3.14) and (3.15), we obtain

$$
\mathbf{A}(\underline{\boldsymbol{\psi}}, \underline{\boldsymbol{\psi}})+\mathbf{C}(\underline{\boldsymbol{\zeta}} ; \underline{\boldsymbol{\psi}}, \underline{\boldsymbol{\psi}}) \geq \frac{\alpha_{\mathbf{A}}}{2}\|\underline{\boldsymbol{\psi}}\|_{\mathbf{X}}^{2}, \quad \underline{\boldsymbol{\psi}} \in \mathbf{X},
$$

that is, the bilinear form $\mathbf{A}(\cdot, \cdot)+\mathbf{C}(\underline{\zeta} ; \cdot, \cdot)$ is elliptic on $\mathbf{X}$. Therefore, applying Lax-Milgram Lemma we obtain that there exists a unique $\underline{\phi} \in \mathbf{X}$ satisfying (3.12). In addition, from (3.9), (3.12) and (3.16), we get

$$
\frac{\alpha_{\mathbf{A}}}{2}\|\underline{\phi}\|_{\mathbf{X}}^{2} \leq \mathbf{A}(\underline{\phi}, \underline{\phi})+\mathbf{C}(\underline{\boldsymbol{\zeta}} ; \underline{\phi}, \underline{\phi})=\mathbf{F}(\underline{\phi}) \leq\|\mathbf{f}\|_{0, \Omega}\left(\kappa_{1}^{2}+\nu^{2}\right)^{1 / 2}\|\underline{\phi}\|_{\mathbf{X}}
$$

which together to (3.13) implies that $\boldsymbol{\phi}$ belongs to $\mathbf{K}$ and concludes the proof.

We now provide the well-posedness of problem (3.3) (or equivalently (2.19)), which corresponds to the main result of this section.

Theorem 3.4 Let $\mathbf{f} \in \mathbf{L}^{2}(\Omega)$ such that

$$
\frac{4 C_{\mathbf{C}}\left(\kappa_{1}^{2}+\nu^{2}\right)^{1 / 2}}{\alpha_{\mathbf{A}}^{2}}\|\mathbf{f}\|_{0, \Omega}<1
$$

Assume that $\kappa_{1}, \kappa_{3}>0$ and $0<\kappa_{2}<2 \nu$. Then, there exists a unique $\underline{\phi} \in \mathbf{X}$ solution to (3.3). In addition, the solution $\phi$ satisfies the continuous dependence result

$$
\|\underline{\phi}\|_{\mathbf{X}} \leq \frac{2\left(\kappa_{1}^{2}+\nu^{2}\right)^{1 / 2}}{\alpha_{\mathbf{A}}}\|\mathbf{f}\|_{0, \Omega}
$$

Proof. First, let us observe that assumption (3.17) ensures that Lemma 3.3 holds true (see assumption (3.13)).

Now, as mentioned before, we make use of the classical Banach's fixed point Theorem to prove that the mapping $\mathcal{J}$ has a unique fixed point in $\mathbf{K}$. In fact, let $\underline{\boldsymbol{\zeta}}_{1}, \underline{\boldsymbol{\zeta}}_{2}, \underline{\phi}_{1}, \underline{\phi}_{2} \in \mathbf{K}$, such that $\underline{\phi}_{1}=\mathcal{J}\left(\underline{\boldsymbol{\zeta}}_{1}\right)$ and $\underline{\phi}_{2}=\mathcal{J}\left(\underline{\boldsymbol{\zeta}}_{2}\right)$. From the definition of $\mathcal{J}$ it follows that

$$
\mathbf{A}\left(\underline{\phi}_{1}-\underline{\phi}_{2}, \underline{\psi}\right)+\mathbf{C}\left(\underline{\boldsymbol{\zeta}}_{1} ; \underline{\phi}_{1}, \underline{\boldsymbol{\psi}}\right)-\mathbf{C}\left(\underline{\boldsymbol{\zeta}}_{2} ; \underline{\phi}_{2}, \underline{\psi}\right)=0, \quad \forall \underline{\boldsymbol{\psi}} \in \mathbf{X} .
$$


Then, by choosing the test function $\underline{\boldsymbol{\psi}}=\underline{\phi}_{1}-\underline{\phi}_{2} \in \mathbf{K}$, and adding and subtracting suitable terms, we arrive at

$$
\mathbf{A}\left(\underline{\phi}_{1}-\underline{\phi}_{2}, \underline{\phi}_{1}-\underline{\phi}_{2}\right)+\mathbf{C}\left(\underline{\boldsymbol{\zeta}}_{2} ; \underline{\phi}_{1}-\underline{\phi}_{2}, \underline{\phi}_{1}-\underline{\phi}_{2}\right)=-\mathbf{C}\left(\underline{\boldsymbol{\zeta}}_{1}-\underline{\boldsymbol{\zeta}}_{2} ; \underline{\phi}_{1}, \underline{\phi}_{1}-\underline{\boldsymbol{\phi}}_{2}\right) .
$$

In this way, we proceed as in the proof of Lemma 3.3, and utilize the continuity of $\mathbf{C}$ and the fact that $\underline{\phi}_{1} \in \mathbf{K}$, to obtain

$$
\left\|\underline{\phi}_{1}-\underline{\phi}_{2}\right\| \mathbf{X} \leq \frac{2 C_{\mathbf{C}}}{\alpha_{\mathbf{A}}}\left\|\underline{\boldsymbol{\phi}}_{1}\right\| \mathbf{X}\left\|\underline{\boldsymbol{\zeta}}_{1}-\underline{\boldsymbol{\zeta}}_{2}\right\| \mathbf{X} \leq \frac{4 C_{\mathbf{C}}\left(\kappa_{1}^{2}+\nu^{2}\right)^{1 / 2}}{\alpha_{\mathbf{A}}^{2}}\|\mathbf{f}\|_{0, \Omega}\left\|\underline{\boldsymbol{\zeta}}_{1}-\underline{\boldsymbol{\zeta}}_{2}\right\|_{\mathbf{X}},
$$

which together to assumption (3.17) implies that $\mathcal{J}$ is a contraction mapping. Hence, applying the Banach's fixed point Theorem we obtain that there exists a unique $\underline{\phi} \in \mathbf{K}$ such that $\mathcal{J}(\underline{\phi})=$ $\underline{\phi}$, or equivalently, there exists a unique $\underline{\phi} \in \mathbf{X}$ solution to (3.3), which satisfies (3.18).

Remark 3.5 We conclude this section by observing that applying Lemma 2.2 and Theorem 3.4, the well-posedness of problem (2.17) is straightforward, where its unique solution given by $\left.(\mathbf{T}, \mathbf{u})=\left(\mathbf{T}_{0}-\frac{1}{n|\Omega|}(\operatorname{tr}(\mathbf{u} \otimes \mathbf{u}), 1)_{\Omega} \mathrm{I}\right), \mathbf{u}\right) \in \mathbb{H}(\operatorname{div} ; \Omega) \times \mathbf{H}^{1}(\Omega)$.

\section{The Galerkin scheme}

In this section we introduce and study the Galerkin scheme of problem (3.3). As we will see in the subsections, the analysis of the corresponding discrete problem follows straightforwardly by adapting the results obtained for the continuous problem to the discrete case.

\subsection{Discrete problem}

We start by introducing the generic finite dimensional subspaces

$$
\mathbf{H}_{h}(\operatorname{div}, \Omega) \subseteq \mathbf{H}(\operatorname{div}, \Omega), \quad \mathrm{H}_{h}^{1}(\Omega) \subseteq \mathrm{H}^{1}(\Omega),
$$

and the discrete spaces

$$
\begin{aligned}
\mathbb{H}_{h} & :=\left\{\mathbf{S}_{h} \in \mathbb{H}(\mathbf{d i v} ; \Omega): \mathbf{c}^{t} \mathbf{S}_{h} \in \mathbf{H}_{h}(\operatorname{div} ; \Omega) \quad \forall \mathbf{c} \in \mathbb{R}^{n}\right\}, \\
\mathbb{H}_{h, 0} & :=\mathbb{H}_{h} \cap \mathbb{H}_{0}(\operatorname{div} ; \Omega), \\
\mathbf{H}_{h}^{1} & :=\left[\mathrm{H}_{h}^{1}(\Omega)\right]^{n} .
\end{aligned}
$$

We observe that in order to have meaningful subspace $\mathbb{H}_{h, 0}$, we need to be able to eliminate multiples of the identity matrix from $\mathbb{H}_{h}$. This request is certainly satisfied if we assume that:

$$
\left[P_{0}(\Omega)\right]^{n \times n} \subseteq \mathbb{H}_{h} .
$$

Then, defining the global discrete space and unknown

$$
\underline{\phi}_{h}:=\left(\mathbf{T}_{h, 0}, \mathbf{u}_{h}\right) \in \mathbf{X}_{h}:=\mathbb{H}_{h, 0} \times \mathbf{H}_{h}^{1},
$$

the discrete problem reads: Find $\underline{\phi}_{h} \in \mathbf{X}_{h}$, such that

$$
\mathbf{A}\left(\underline{\phi}_{h}, \underline{\boldsymbol{\psi}}_{h}\right)+\mathbf{C}\left(\underline{\boldsymbol{\phi}}_{h} ; \underline{\boldsymbol{\phi}}_{h}, \underline{\boldsymbol{\psi}}_{h}\right)=\mathbf{F}\left(\underline{\boldsymbol{\psi}}_{h}\right) \quad \forall \underline{\boldsymbol{\psi}}_{h}:=\left(\mathbf{S}_{h}, \mathbf{v}_{h}\right) \in \mathbf{X}_{h},
$$

with $\mathbf{A}, \mathbf{C}$ and $\mathbf{F}$ defined in (3.1) and (3.2). 
Remark 4.1 It is not difficult to see that, similarly to the continuous case, $\left(\mathbf{T}_{h, 0}, \mathbf{u}_{h}\right)$ is a solution of problem (4.4), if and only if, $\left(\mathbf{T}_{h, 0}-\frac{1}{n|\Omega|}\left(\operatorname{tr}\left(\mathbf{u}_{h} \otimes \mathbf{u}_{h}\right), 1\right)_{\Omega} \mathrm{I}, \mathbf{u}_{h}\right)$ is a solution of the discrete version of problem (2.17): Find $\underline{\boldsymbol{\phi}}_{h}:=\left(\mathbf{T}_{h}, \mathbf{u}_{h}\right) \in \mathbb{H}_{h} \times \mathbf{H}_{h}^{1}$, such that $\left(\operatorname{tr}\left(\mathbf{T}_{\mathbf{h}}\right)+\operatorname{tr}\left(\mathbf{u}_{h} \otimes\right.\right.$ $\left.\left.\mathbf{u}_{h}\right), 1\right)_{\Omega}=0$ and

$$
\mathbf{A}\left(\underline{\tilde{\phi}}_{h}, \underline{\boldsymbol{\psi}}_{h}\right)+\mathbf{C}\left(\underline{\tilde{\phi}}_{h} ; \underline{\tilde{\phi}}_{h}, \underline{\boldsymbol{\psi}}_{h}\right)=\mathbf{F}\left(\underline{\boldsymbol{\psi}}_{h}\right) \quad \forall \underline{\boldsymbol{\psi}}_{h}:=\left(\mathbf{S}_{h}, \mathbf{v}_{h}\right) \in \mathbb{H}_{h} \times \mathbf{H}_{h}^{1} .
$$

According to this, provided the solution $\underline{\phi}_{h}=\left(\mathbf{T}_{h, 0}, \mathbf{u}_{h}\right) \in \mathbf{X}_{h}$ of problem (4.4), the approximate solution of problem (2.17) is given by $\left(\overline{\mathbf{T}}_{h}, \mathbf{u}_{h}\right) \in \mathbb{H}_{h} \times \mathbf{H}_{h}^{1}$, where the tensor $\mathbf{T}_{h}$ is computed by the formula:

$$
\mathbf{T}_{h}=\mathbf{T}_{h, 0}-\frac{1}{n|\Omega|}\left(\operatorname{tr}\left(\mathbf{u}_{h} \otimes \mathbf{u}_{h}\right), 1\right)_{\Omega} \mathrm{I} .
$$

This formula will be employed later to approximate further variables of interest.

Remark 4.2 Notice that the only requirement on the finite element subspaces defined in (4.2) is (4.3), and as we will see later, no further requirements need to be added. This is one of the key features of our approach; the freedom of choosing discrete spaces.

\subsection{Well-posedness of the discrete problem}

We begin by observing that the boundedness of the forms $\mathbf{A}, \mathbf{C}$, and the functional $\mathbf{F}$, namely (3.6), (3.7) and (3.9), is clearly inherited from the continuous case. In addition, the following lemma holds.

Lemma 4.3 Assume that $\kappa_{1}, \kappa_{3}>0$ and $0<\kappa_{2}<2 \nu$. Then there exists $\alpha_{\mathbf{A}}>0$, such that

$$
\mathbf{A}\left(\underline{\boldsymbol{\psi}}_{h}, \underline{\boldsymbol{\psi}}_{h}\right) \geq \alpha_{\mathbf{A}}\left\|\underline{\boldsymbol{\psi}}_{h}\right\|_{\mathbf{X}}^{2} \quad \forall \underline{\boldsymbol{\psi}}_{h} \in \mathbf{X}_{h}
$$

Proof. The proof follows by applying the same arguments utilized in the proof of Lemma 3.1. We omit further details.

Now, analogously to the continuous case, in what follows we apply the classical Banach's fixed point theorem to prove well-posedness of problem (4.4). To that end, we introduce the finite dimensional bounded set

$$
\mathbf{K}_{h}:=\left\{\underline{\boldsymbol{\psi}}_{h} \in \mathbf{X}_{h}:\left\|\underline{\boldsymbol{\psi}}_{h}\right\| \mathbf{x} \leq \frac{2\left(\kappa_{1}^{2}+\nu^{2}\right)^{1 / 2}}{\alpha_{\mathbf{A}}}\|\mathbf{f}\|_{0, \Omega}\right\},
$$

and the discrete version of $\mathcal{J}$ :

$$
\mathcal{J}_{h}: \mathbf{K}_{h} \rightarrow \mathbf{K}_{h}, \quad \underline{\boldsymbol{\zeta}}_{h} \rightarrow \mathcal{J}_{h}\left(\underline{\boldsymbol{\zeta}}_{h}\right)=\underline{\phi}_{h},
$$

with $\underline{\boldsymbol{\zeta}}_{h}$ and $\underline{\phi}_{h}$ satisfying

$$
\mathbf{A}\left(\underline{\phi}_{h}, \underline{\boldsymbol{\psi}}_{h}\right)+\mathbf{C}\left(\underline{\boldsymbol{\zeta}}_{h} ; \underline{\phi}_{h}, \underline{\boldsymbol{\psi}}_{h}\right)=\mathbf{F}\left(\underline{\boldsymbol{\psi}}_{h}\right) \quad \forall \underline{\boldsymbol{\psi}}_{h} \in \mathbf{X}_{h} .
$$

The following Lemma establishes that the operator $\mathcal{J}_{h}$ is well-defined. 
Lemma 4.4 Assume that

$$
\frac{4 C_{\mathbf{C}}\left(\kappa_{1}^{2}+\nu^{2}\right)^{1 / 2}}{\alpha_{\mathbf{A}}^{2}}\|\mathbf{f}\|_{0, \Omega} \leq 1,
$$

with $C_{\mathbf{C}}$ and $\alpha_{\mathbf{A}}$ being the constants in (3.7) and (3.8), respectively. Assume further that $\kappa_{1}, \kappa_{3}>0$ and $0<\kappa_{2}<2 \nu$. Then, given $\underline{\boldsymbol{\zeta}}_{h} \in \mathbf{K}_{h}$, there exists a unique $\underline{\phi}_{h} \in \mathbf{K}_{h}$ such that $\mathcal{J}_{h}\left(\underline{\boldsymbol{\zeta}}_{h}\right)=\underline{\phi}_{h}$.

Proof. Similarly to the proof of Lemma 3.3, we let $\underline{\boldsymbol{\zeta}}_{h} \in \mathbf{K}_{h}$, assume inequality (4.10), and apply Lemma 4.3, to obtain

$$
\mathbf{A}\left(\underline{\boldsymbol{\psi}}_{h}, \underline{\boldsymbol{\psi}}_{h}\right)+\mathbf{C}\left(\underline{\boldsymbol{\zeta}}_{h} ; \underline{\boldsymbol{\psi}}_{h}, \underline{\boldsymbol{\psi}}_{h}\right) \geq \frac{\alpha_{\mathbf{A}}}{2}\left\|_{\underline{\boldsymbol{\psi}}_{h}}\right\|_{\mathbf{X}}^{2}, \quad \forall \underline{\boldsymbol{\psi}}_{h} \in \mathbf{X}_{h}
$$

that is, the bilinear form $\mathbf{A}(\cdot, \cdot)+\mathbf{C}\left(\underline{\boldsymbol{\zeta}}_{h} ; \cdot, \cdot\right)$ is elliptic on $\mathbf{X}_{h}$. This inequality, together to (3.7), (3.9), and assumption (4.10) imply the result.

Finally, we establish the well-posedness of problem (4.4).

Theorem 4.5 Let $\mathbf{f} \in \mathbf{L}^{2}(\Omega)$ such that

$$
\frac{4 C_{\mathbf{C}}\left(\kappa_{1}^{2}+\nu^{2}\right)^{1 / 2}}{\alpha_{\mathbf{A}}^{2}}\|\mathbf{f}\|_{0, \Omega}<1
$$

Assume that $\kappa_{1}, \kappa_{3}>0$ and $0<\kappa_{2}<2 \nu$. Then, there exists a unique $\underline{\phi}_{h} \in \mathbf{X}_{h}$ solution to (4.4). In addition, the solution $\underline{\phi}_{h}$ satisfies the continuous dependence result

$$
\left\|\underline{\phi}_{h}\right\| \mathbf{X} \leq \frac{2\left(\kappa_{1}^{2}+\nu^{2}\right)^{1 / 2}}{\alpha_{\mathbf{A}}}\|\mathbf{f}\|_{0, \Omega}
$$

Proof. Let us observe that $\mathcal{J}_{h}\left(\underline{\phi}_{h}\right)=\underline{\phi}_{h}$ if and only if $\underline{\phi}_{h}$ is the unique solution of problem (4.4) satisfying (4.13). Then, analogously to the proof of Theorem 3.4, it suffices to prove that $\mathcal{J}_{h}$ is a contraction mapping in $\mathbf{K}_{h}$, which follows straightforwardly from the proof of Theorem 3.4.

\subsection{Cea's estimate}

Now, we derive the corresponding Cea's estimate of our Galerkin scheme (4.4). This result is established in the following theorem.

Theorem 4.6 Let $\underline{\phi}:=\left(\mathbf{T}_{0}, \mathbf{u}\right) \in \mathbf{X}$ and $\underline{\phi}_{h}:=\left(\mathbf{T}_{h, 0}, \mathbf{u}_{h}\right) \in \mathbf{X}_{h}$ be the unique solutions of the continuous and discrete problems (3.3) and (4.4), respectively, with $\mathbf{f} \in \mathbf{L}^{2}(\Omega)$ satisfying (4.12). Assume that $\kappa_{1}, \kappa_{3}>0$ and $0<\kappa_{2}<2 \nu$. Then, there exists $C_{c e a}>0$, independent of $h$, such that

$$
\left\|\underline{\phi}-\underline{\phi}_{h}\right\| \mathrm{x} \leq C_{c e a} \inf _{\underline{\boldsymbol{\psi}}_{h} \in \mathrm{X}_{h}}\left\|\underline{\phi}-\underline{\psi}_{h}\right\| \mathrm{X} .
$$

Proof. In order to simplify the subsequent analysis, we write $\mathbf{e}_{\underline{\phi}}=\underline{\phi}-\underline{\phi}_{h}$. As usual, for any $\underline{\hat{\boldsymbol{\psi}}}_{h}:=\left(\hat{\mathbf{S}}_{h}, \hat{\mathbf{v}}_{h}\right) \in \mathbf{X}_{h}$, we shall decompose this error into

$$
\mathbf{e}_{\underline{\phi}}=\boldsymbol{\xi}_{\underline{\phi}}+\chi_{\underline{\phi}}=\left(\underline{\phi}-\underline{\hat{\psi}}_{h}\right)+\left(\underline{\hat{\psi}}_{h}-\underline{\phi}_{h}\right) .
$$


Now, subtracting equations (3.3) and (4.4), we easily get the Galerkin orthogonality property

$$
\mathbf{A}\left(\mathbf{e}_{\underline{\phi}}, \underline{\boldsymbol{\psi}}_{h}\right)+\left[\mathbf{C}\left(\underline{\boldsymbol{\phi}} ; \underline{\boldsymbol{\phi}}, \underline{\boldsymbol{\psi}}_{h}\right)-\mathbf{C}\left(\underline{\boldsymbol{\phi}}_{h} ; \underline{\boldsymbol{\phi}}_{h}, \underline{\boldsymbol{\psi}}_{h}\right)\right]=0 \quad \forall \underline{\boldsymbol{\psi}}_{h} \in \mathbf{X}_{h}
$$

In turn, adding and subtracting suitable terms we get

$$
\mathbf{A}\left(\mathbf{e}_{\underline{\phi}}, \underline{\boldsymbol{\psi}}_{h}\right)+\left[\mathbf{C}\left(\mathbf{e}_{\underline{\phi}} ; \underline{\boldsymbol{\phi}}_{h}, \underline{\boldsymbol{\psi}}_{h}\right)+\mathbf{C}\left(\underline{\boldsymbol{\phi}} ; \underline{\mathbf{e}}_{\boldsymbol{\phi}}, \underline{\boldsymbol{\psi}}_{h}\right)\right]=0 \quad \forall \underline{\boldsymbol{\psi}}_{h} \in \mathbf{X}_{h}
$$

which implies

$$
\begin{aligned}
\mathbf{A}\left(\chi_{\underline{\phi}}, \underline{\psi}_{h}\right)+\mathbf{C}\left(\chi_{\underline{\phi}} ; \underline{\phi}_{h}, \underline{\psi}_{h}\right)= & -\mathbf{A}\left(\underline{\boldsymbol{\xi}}_{\underline{\phi}}, \underline{\boldsymbol{\psi}}_{h}\right)-\mathbf{C}\left(\underline{\boldsymbol{\xi}}_{\underline{\phi}} ; \underline{\phi}_{h}, \underline{\boldsymbol{\psi}}_{h}\right)-\mathbf{C}\left(\underline{\phi} ; \underline{\chi}_{\underline{\phi}}, \underline{\boldsymbol{\psi}}_{h}\right) \\
& -\mathbf{C}\left(\underline{\phi} ; \underline{\boldsymbol{\xi}}_{\underline{\phi}}, \underline{\boldsymbol{\psi}}_{h}\right),
\end{aligned}
$$

for all $\underline{\boldsymbol{\psi}}_{h} \in \mathbf{X}_{h}$. In particular, for $\underline{\boldsymbol{\psi}}_{h}=\chi_{\underline{\phi}}$, from (4.11), and the continuity of $\mathbf{A}$ and $\mathbf{C}$, we obtain

$$
\frac{\alpha_{\mathbf{A}}}{2}\left\|\chi_{\underline{\phi}}\right\|_{\mathbf{X}} \leq\left(C_{\mathbf{A}}+C_{\mathbf{C}}\left\|\underline{\phi}_{h}\right\| \mathbf{X}+C_{\mathbf{C}}\|\underline{\phi}\|_{\mathbf{X}}\right)\left\|\boldsymbol{\xi}_{\underline{\phi}}\right\|_{\mathbf{X}}+C_{\mathbf{C}}\|\underline{\phi}\|_{\mathbf{X}}\left\|\chi_{\underline{\phi}}\right\|_{\mathbf{X}}
$$

which together to the fact that $\underline{\phi} \in \mathbf{K}$ and $\underline{\phi}_{h} \in \mathbf{K}_{h}$, yield

$$
\left(1-\frac{4 C_{\mathbf{C}}\left(\kappa_{1}^{2}+\nu^{2}\right)^{1 / 2}}{\alpha_{\mathbf{A}}^{2}}\|\mathbf{f}\|_{0, \Omega}\right)\left\|\chi_{\underline{\phi}}\right\|_{\mathbf{X}} \leq\left(\frac{2 C_{\mathbf{A}}}{\alpha_{\mathbf{A}}}+\frac{8 C_{\mathbf{C}}\left(\kappa_{1}^{2}+\nu^{2}\right)^{1 / 2}}{\alpha_{\mathbf{A}}^{2}}\|\mathbf{f}\|_{0, \Omega}\right)\left\|\boldsymbol{\xi}_{\underline{\phi}}\right\|_{\mathbf{X}}
$$

In this way, thanks to assumption (4.12), from (4.15), (4.17) and the triangle inequality we obtain that there exists $C_{c e a}>0$, independent of $h$, such that

$$
\left\|\underline{\phi}-\underline{\phi}_{h}\right\|_{\mathbf{X}}=\left\|\mathbf{e}_{\underline{\phi}}\right\|_{\mathbf{X}} \leq\left\|\chi_{\underline{\phi}}\right\|_{\mathbf{X}}+\left\|\boldsymbol{\xi}_{\underline{\phi}}\right\|_{\mathbf{X}} \leq C_{c e a}\left\|\boldsymbol{\xi}_{\underline{\phi}}\right\|_{\mathbf{X}}=C_{c e a}\left\|\underline{\phi}-\underline{\hat{\psi}}_{h}\right\|_{\mathbf{X}},
$$

for all $\underline{\hat{\psi}}_{h} \in \mathbf{X}_{h}$, which concludes the proof.

\subsection{Computing further variables of interest}

As mentioned before, one of the advantages of solving fluid flow problems through peudostresstype formulations, is the possibility of approximating further variables of interest. In fact, provided the solution $\left(\mathbf{T}_{h, 0}, \mathbf{u}_{h}\right) \in \mathbf{X}_{h}$ of problem (4.4), we first recall that the solution of problem $(2.17)$ can be easily approximated by $\left(\mathbf{T}_{h}, \mathbf{u}_{h}\right):=\left(\mathbf{T}_{h, 0}-\frac{1}{n|\Omega|}\left(\operatorname{tr}\left(\mathbf{u}_{h} \otimes \mathbf{u}_{h}\right), 1\right)_{\Omega} \mathrm{I}, \mathbf{u}_{h}\right)$ (see remark 4.1), where $\mathbf{T}_{h} \in \mathbb{H}_{h}$ turns to be the approximation of tensor $\mathbf{T}$ defined in (2.4). According to this, and recalling the formulae provided in (2.6) and (2.11), the pressure $p$, the stress $\tilde{\boldsymbol{\sigma}}:=\nu\left(\nabla \mathbf{u}+(\nabla \mathbf{u})^{t}\right)-p \mathrm{I}$, the velocity gradient $\mathbf{G}=\nabla \mathbf{u}$, and the vorticity $\boldsymbol{\omega}:=$ $\frac{1}{2}\left(\nabla \mathbf{u}-\nabla \mathbf{u}^{t}\right)$ can be approximated as follows:

$$
\begin{aligned}
p_{h} & =-\frac{1}{n}\left(\operatorname{tr}\left(\mathbf{T}_{h}\right)+\operatorname{tr}\left(\mathbf{u}_{h} \otimes \mathbf{u}_{h}\right)\right) \\
\tilde{\boldsymbol{\sigma}}_{h} & =\mathbf{T}_{h}^{d}+\left(\mathbf{u}_{h} \otimes \mathbf{u}_{h}\right)^{d}+\mathbf{T}_{h}^{t}+\mathbf{u}_{h} \otimes \mathbf{u}_{h}, \\
\mathbf{G}_{h} & =\frac{1}{\nu}\left(\mathbf{T}_{h}^{d}+\left(\mathbf{u}_{h} \otimes \mathbf{u}_{h}\right)^{d}\right), \\
\boldsymbol{\omega}_{h} & =\frac{1}{2 \nu}\left(\mathbf{T}_{h}-\mathbf{T}_{h}^{t}\right) .
\end{aligned}
$$

The following corollary establishes the corresponding approximation result for this postprocessing procedure. 
Corollary 4.7 Assume that the hypotheses of Theorem 4.6 hold true. Let $\phi:=\left(\mathbf{T}_{0}, \mathbf{u}\right) \in \mathbf{X}$ and $\underline{\phi}_{h}:=\left(\mathbf{T}_{h, 0}, \mathbf{u}_{h}\right) \in \mathbf{X}_{h}$ be the unique solutions of the continuous and discrete problems (3.3) and (4.4), respectively. Let $\mathbf{T}_{h}=\mathbf{T}_{h, 0}-\frac{1}{n|\Omega|}\left(\operatorname{tr}\left(\mathbf{u}_{h} \otimes \mathbf{u}_{h}\right), 1\right)_{\Omega} \mathrm{I}$, and let $p_{h}, \tilde{\boldsymbol{\sigma}}_{h}, \mathbf{G}_{h}$ and $\boldsymbol{\omega}_{h}$ given by (4.18). Then there exists $\hat{C}>0$, independent of $h$, such that

$$
\left\|p-p_{h}\right\|_{0, \Omega}+\left\|\tilde{\boldsymbol{\sigma}}-\tilde{\boldsymbol{\sigma}}_{h}\right\|_{0, \Omega}+\left\|\mathbf{G}-\mathbf{G}_{h}\right\|_{0, \Omega}+\left\|\boldsymbol{\omega}-\boldsymbol{\omega}_{h}\right\|_{0, \Omega} \leq \hat{C} \inf _{\underline{\boldsymbol{\psi}}_{h} \in \mathbf{X}_{h}}\left\|\underline{\boldsymbol{\phi}}-\underline{\boldsymbol{\psi}}_{h}\right\| \mathbf{x} .
$$

Proof. Since $H^{1}$ is continuously embedded in $L^{4}$, and recalling that $\underline{\phi} \in \mathbf{K}$ and $\underline{\phi}_{h} \in \mathbf{K}_{h}$, it is not difficult to see that

$$
\begin{aligned}
\left\|\mathbf{u} \otimes \mathbf{u}-\mathbf{u}_{h} \otimes \mathbf{u}_{h}\right\|_{0, \Omega} & \leq\left\|\left(\mathbf{u}-\mathbf{u}_{h}\right) \otimes \mathbf{u}\right\|_{0, \Omega}+\left\|\mathbf{u}_{h} \otimes\left(\mathbf{u}-\mathbf{u}_{h}\right)\right\|_{0, \Omega} \\
& \leq C\left\{\left\|\left(\mathbf{u}-\mathbf{u}_{h}\right)\right\|_{1, \Omega}\|\mathbf{u}\|_{1, \Omega}+\left\|\left(\mathbf{u}-\mathbf{u}_{h}\right)\right\|_{1, \Omega}\left\|\mathbf{u}_{h}\right\|_{1, \Omega}\right\} \\
& \leq 2 C\left\|\left(\mathbf{u}-\mathbf{u}_{h}\right)\right\|_{1, \Omega}\|\mathbf{f}\|_{0, \Omega} .
\end{aligned}
$$

Then, the result follows from (4.14), (4.20), and equations (2.6), (2.11), (2.18) and (4.18).

\subsection{A particular choice of discrete spaces}

We now specify a concrete example of finite element subspaces for our Galerkin scheme (4.4). To this end we let $\mathcal{T}_{h}$ be a regular family of triangulations of the polyhedral region $\bar{\Omega}$ by triangles $K$ in $\mathbb{R}^{2}$ or tetrahedra in $\mathbb{R}^{3}$ of diameter $h_{K}$ such that $\bar{\Omega}=\cup\left\{K: K \in \mathcal{T}_{h}\right\}$ and define $h:=\max \left\{h_{K}: K \in \mathcal{T}_{h}\right\}$. Now, given an integer $l \geq 0$ and a subset $S$ of $\mathbb{R}^{n}$, we denote by $P_{l}(S)$ the space of polynomials of total degree at most $l$ defined on $S$.

For each integer $k \geq 0$ and for each $K \in \mathcal{T}_{h}$, we define the local Raviart-Thomas space of order $k$ (see, for instance [4]):

$$
\mathbf{R T}_{k}(K):=\left[P_{k}(K)\right]^{n} \oplus P_{k}(K) \mathbf{x}
$$

where $\mathbf{x}:=\left(x_{1}, \ldots, x_{n}\right)^{t}$ is a generic vector of $\mathbb{R}^{n}$. Then, we specify the discrete spaces in (4.1) by:

$$
\begin{gathered}
\mathbf{H}_{h}(\operatorname{div} ; \Omega):=\left\{T \in \mathbf{H}(\operatorname{div} ; \Omega):\left.T\right|_{K} \in \mathbf{R T}_{k}(K), \quad \forall K \in \mathcal{T}_{h}\right\}, \\
\mathrm{H}_{h}^{1}(\Omega):=\left\{v \in C(\bar{\Omega}):\left.\quad v\right|_{K} \in P_{k+1}(K), \quad \forall K \in \mathcal{T}_{h}\right\} .
\end{gathered}
$$

It is well known that these subspaces satisfy the following approximation properties (see, e.g. $[9],[18],[27])$ :

For each $s>0$ and for each $T \in \mathbf{H}^{s}(\Omega)$, with $\operatorname{div} T \in \mathrm{H}^{s}(\Omega)$, there exists $T_{h} \in \mathbf{H}_{h}$, such that

$$
\left\|T-T_{h}\right\|_{\operatorname{div}, \Omega} \leq C h^{\min \{s, k+1\}}\left\{\|T\|_{s, \Omega}+\|\operatorname{div} T\|_{s, \Omega}\right\} .
$$

For each $s>0$ and for each $v \in \mathrm{H}^{s+1}(\Omega)$ there exists $v_{h} \in \mathrm{H}_{h}^{1}(\Omega)$ such that

$$
\left\|v-v_{h}\right\|_{1, \Omega} \leq C h^{\min \{s, k+1\}}\|v\|_{s+1, \Omega} .
$$

As a consequence of the above we can establish the convergence result of our Galerkin scheme (4.4) for this particular choice of spaces. 
Theorem 4.8 Let $\mathbf{X}_{h}=\mathbb{H}_{h, 0} \times \mathbf{H}_{h}^{1}$ defined in terms of the finite element spaces (4.21). Let $\underline{\phi}:=\left(\mathbf{T}_{0}, \mathbf{u}\right) \in \mathbf{X}$ and $\underline{\phi}_{h}:=\left(\mathbf{T}_{h, 0}, \mathbf{u}_{h}\right) \in \mathbf{X}_{h}$ be the unique solutions of the continuous and discrete problems (3.3) and (4.4), respectively, with $\mathbf{f} \in \mathbf{L}^{2}(\Omega)$ satisfying (4.12). Assume that $\kappa_{1}, \kappa_{3}>0$ and $0<\kappa_{2}<2 \nu$. Assume further that $\mathbf{T}_{0} \in \mathbb{H}^{s}(\Omega)$, $\operatorname{div} \mathbf{T}_{0} \in \mathbf{H}^{s}(\Omega)$, and $\mathbf{u} \in$ $\mathbf{H}^{s+1}(\Omega)$, for some $s>0$. Then there exists $C_{\text {rate }}>0$, independent of $h$, such that

$$
\left\|\underline{\phi}-\underline{\phi}_{h}\right\| \mathbf{X} \leq C_{\text {rate }} h^{\min \{s, k+1\}}\left\{\left\|\mathbf{T}_{0}\right\|_{s, \Omega}+\left\|\operatorname{div} \mathbf{T}_{0}\right\|_{s, \Omega}+\|\mathbf{u}\|_{s+1, \Omega}\right\}
$$

Proof. The result is a straightforward application of Theorem 4.6, and properties (4.22) and (4.23).

We now provide the following corollary establishing the rate of convergence for the postprocessing procedure introduced in (4.18).

Corollary 4.9 Let $\mathbf{X}_{h}=\mathbb{H}_{h, 0} \times \mathbf{H}_{h}^{1}$ defined in terms of the finite element spaces (4.21). Let $\underline{\phi}:=\left(\mathbf{T}_{0}, \mathbf{u}\right) \in \mathbf{X}$ and $\underline{\phi}_{h}:=\left(\mathbf{T}_{h, 0}, \mathbf{u}_{h}\right) \in \mathbf{X}_{h}$ be the unique solutions of the continuous and discrete problems (3.3) and (4.4), respectively, with $\mathbf{f} \in \mathbf{L}^{2}(\Omega)$ satisfying (4.12). Assume that $\kappa_{1}, \kappa_{3}>0$ and $0<\kappa_{2}<2 \nu$. Assume further that $\mathbf{T}_{0} \in \mathbb{H}^{s}(\Omega)$, $\operatorname{div} \mathbf{T}_{0} \in \mathbf{H}^{s}(\Omega)$, and $\mathbf{u} \in$ $\mathbf{H}^{s+1}(\Omega)$, for some $s>0$. Let $\mathbf{T}_{h}=\mathbf{T}_{h, 0}-\frac{1}{n|\Omega|}\left(\operatorname{tr}\left(\mathbf{u}_{h} \otimes \mathbf{u}_{h}\right), 1\right)_{\Omega} \mathbf{I}$, and let $p_{h}, \tilde{\boldsymbol{\sigma}}_{h}, \mathbf{G}_{h}$ and $\boldsymbol{\omega}_{h}$ given by (4.18). Then there exists $\tilde{C}>0$, independent of $h$, such that

$$
\begin{array}{r}
\left\|p-p_{h}\right\|_{0, \Omega}+\left\|\tilde{\boldsymbol{\sigma}}-\tilde{\boldsymbol{\sigma}}_{h}\right\|_{0, \Omega}+\left\|\mathbf{G}-\mathbf{G}_{h}\right\|_{0, \Omega}+\left\|\boldsymbol{\omega}-\boldsymbol{\omega}_{h}\right\|_{0, \Omega} \\
\leq \tilde{C} h^{\min \{s, k+1\}}\left\{\left\|\mathbf{T}_{0}\right\|_{s, \Omega}+\left\|\operatorname{div} \mathbf{T}_{0}\right\|_{s, \Omega}+\|\mathbf{u}\|_{s+1, \Omega}\right\} .
\end{array}
$$

Proof. The result is a direct application of Theorem 4.8 and Corollary 4.7.

\section{$5 \quad$ Iterative methods}

In this section we introduce and analyze two iterative methods to compute the solution of the discrete nonlinear system (4.4), namely a fixed point iterative method and the classical Newton's method. For simplicity of notation, in what follows we present the algorithms in the continuous case since, at discrete level, the analysis can be exactly replicated from the continuous case. In addition we shall observe that the convergence results of the iterative methods at discrete level are totally independent of the mesh, due to the fact that they are established by using the properties of the forms $\mathbf{A}$ and $\mathbf{C}$ at the continuous level.

\subsection{Fixed-point strategy}

Our first strategy to solve the nonlinear problem (3.3) is given by a fixed point iteration written in the following form: Given $\underline{\phi}^{0} \in \mathbf{X}$, for $m \geq 1$, find $\underline{\phi}^{m} \in \mathbf{X}$ such that

$$
\mathbf{A}\left(\underline{\phi}^{m}, \underline{\psi}\right)+\mathbf{C}\left(\underline{\phi}^{m-1} ; \underline{\phi}^{m}, \underline{\psi}\right)=\mathbf{F}(\underline{\boldsymbol{\psi}}) \quad \forall \underline{\boldsymbol{\psi}} \in \mathbf{X} .
$$

Notice that this strategy is nothing but the classical Picard-type iteration to find the unique fixed point of operator $\mathcal{J}$ given in (3.11) (or $\mathcal{J}_{h}$ given in (4.8)) which has been proved that is a contraction mapping in $\mathbf{K}$ (or $\mathbf{K}_{h}$ for the discrete problem). As a consequence, the convergence of this iterative process is ensured by Theorem 3.4 (respectively Theorem 4.5 for the discrete problem). This result is provided next. Its proof is straightforward. 
Proposition 5.1 Let $\mathbf{f} \in \mathbf{L}^{2}(\Omega)$ such that (3.17) holds. Assume that $\kappa_{1}, \kappa_{3}>0$ and $0<\kappa_{2}<$ $2 \nu$. Then, given an initial guess $\underline{\phi}^{0}$, satisfying

$$
\left\|\underline{\phi}^{0}\right\|_{\mathbf{X}} \leq \frac{2\left(\kappa_{1}^{2}+\nu^{2}\right)^{1 / 2}}{\alpha_{\mathbf{A}}}\|\mathbf{f}\|_{0, \Omega}
$$

the sequence $\underline{\phi}^{n}$ given by (5.1) converges to the unique solution $\underline{\phi}$ of problem (3.3) when $n$ tends to infinity, and

$$
\|\underline{\phi}\| \mathbf{X} \leq \frac{2\left(\kappa_{1}^{2}+\nu^{2}\right)^{1 / 2}}{\alpha_{\mathbf{A}}}\|\mathbf{f}\|_{0, \Omega}
$$

\subsection{Newton's method}

Now we present the well known Newton's method to solve the nonlinear problem (3.3). Before describing this strategy applied to our problem, we first recall this method in an abstract framework and introduce a classical theorem ensuring its convergence, namely, the Kantorovich Theorem. To do this, we let $X$ and $Y$ be two Banach spaces and $x_{0}$ a given element in $X$. In addition, we let $B_{R}$ and $\bar{B}_{r}$ be the bounded sets defined by

$$
B_{R}:=\left\{x \in X:\left\|x-x_{0}\right\|_{X}<R\right\}
$$

and

$$
\bar{B}_{r}:=\left\{x \in X:\left\|x-x_{0}\right\|_{X} \leq r\right\},
$$

where $R$ and $r$ are positive constants satisfying $0<r<R$. Let us assume that $B_{R}$ contains a zero of an operator $P: B_{R} \subset X \rightarrow Y$, i.e., a point $x^{*} \in B_{R}$ such that $P\left(x^{*}\right)=0$, and that $P$ has a continuous derivative in $B_{R}$. Then the Newton's method is described as follows:

Newton's method: Given an initial approximation $x^{0} \in B_{R}$ of $x^{*}$, compute:

$$
x^{m}=x^{m-1}-\left[P^{\prime}\left(x^{m-1}\right)\right]^{-1}\left(P\left(x^{m-1}\right)\right) \quad \text { for } \quad m \geq 1,
$$

assuming that $\left[P^{\prime}\left(x^{m-1}\right)\right]^{-1}$ exists.

The following theorem establishes the convergence of this method (for details, see Theorem 5 in [3] or Theorem 6 (1.XVIII) in [30, p. 708]).

Theorem 5.2 (Kantorovich Theorem) Let $P$ be defined on $B_{R} \subset X$ with continuous second derivative in $\bar{B}_{r}$. Moreover assume that

(1) there exists the continuous linear operator $\left[P^{\prime}\left(x^{0}\right)\right]^{-1}$,

(2) there exists a positive constant $K_{1}>0:\left\|\left[P^{\prime}\left(x^{0}\right)\right]^{-1}\left(P\left(x^{0}\right)\right)\right\| \leq K_{1}$,

(3) there exists a positive constant $K_{2}>0:\left\|\left[P^{\prime}\left(x^{0}\right)\right]^{-1} P^{\prime \prime}(x)\right\| \leq K_{2}$, for all $x \in \bar{B}_{r}$.

If

$$
K_{3}=K_{1} K_{2} \leq \frac{1}{2}
$$

and the radious $r$ of $\bar{B}_{r}$ satisfies

$$
r \geq r_{0}=\frac{1-\sqrt{1-2 K_{3}}}{K_{3}} K_{1},
$$


then, there exists a zero $x^{*}$ of $P$ to which the Newton's iteration converges. In this case,

$$
\left\|x^{*}-x^{0}\right\|_{X} \leq r_{0}
$$

Furthermore, if for $K_{3}<1 / 2$

$$
r<r_{1}=\frac{1+\sqrt{1-2 K_{3}}}{K_{3}} K_{1}
$$

or for $K_{3}=1 / 2$

$$
r \leq r_{1}
$$

the solution $x^{*}$ is unique in the set $\bar{B}_{r}$.

The rate of convergence of the Newton's method is given by

$$
\left\|x^{*}-x^{m}\right\|_{X} \leq \frac{1}{2^{m}}\left(2 K_{3}\right)^{2^{m}} \frac{K_{1}}{K_{3}}, \quad m \geq 0 .
$$

In what follows, we apply the theory described above to derive and analyze the Newton's method associated to problem (3.3). To do so, we let $\underline{\boldsymbol{\zeta}}$ be a fixed element in $\mathbf{X}$, and introduce the linear operator $\mathcal{N}(\underline{\boldsymbol{\zeta}}, \cdot): \mathbf{X} \rightarrow \mathbf{X}^{\prime}$, defined by

$$
\langle\mathcal{N}(\underline{\zeta}, \underline{\phi}), \underline{\psi}\rangle:=\mathbf{A}(\underline{\phi}, \underline{\psi})+\mathbf{C}(\underline{\zeta} ; \underline{\phi}, \underline{\psi}) \quad \forall \underline{\phi}, \underline{\psi} \in \mathbf{X}
$$

and the functional $\mathcal{F} \in \mathbf{X}^{\prime}$

$$
\langle\mathcal{F}, \underline{\psi}\rangle:=\mathbf{F}(\underline{\psi}) \quad \forall \underline{\psi} \in \mathbf{X}
$$

Then, defining the nonlinear operator $\mathcal{P}: \mathbf{X} \rightarrow \mathbf{X}^{\prime}$, as

$$
\mathcal{P}(\underline{\boldsymbol{\psi}}):=\mathcal{N}(\underline{\boldsymbol{\psi}}, \underline{\boldsymbol{\psi}})-\mathcal{F} \quad \forall \underline{\boldsymbol{\psi}} \in \mathbf{X},
$$

we obtain that $\underline{\phi} \in \mathbf{X}$ is the unique solution of (3.3), if and only if,

$$
\langle\mathcal{P}(\underline{\phi}), \underline{\psi}\rangle=\mathbf{A}(\underline{\phi}, \underline{\psi})+\mathbf{C}(\underline{\phi} ; \underline{\phi}, \underline{\psi})-\mathbf{F}(\underline{\psi})=0 \quad \forall \underline{\psi} \in \mathbf{X},
$$

that is, $\mathcal{P}(\underline{\phi})=\underline{\mathbf{0}} \in \mathbf{X}^{\prime}$.

Now, after simple computations, it is not difficult to see that the Gateaux derivative of $\mathcal{P}$, $\mathcal{P}^{\prime}: \mathbf{X} \rightarrow \mathcal{L}\left(\mathbf{X}, \mathbf{X}^{\prime}\right)$, is given by

$$
\left\langle\left(\mathcal{P}^{\prime}(\underline{\phi})\right)(\underline{\boldsymbol{\zeta}}), \underline{\psi}\right\rangle=\mathbf{A}(\underline{\boldsymbol{\zeta}}, \underline{\psi})+\mathbf{C}(\underline{\boldsymbol{\zeta}} ; \underline{\phi}, \underline{\psi})+\mathbf{C}(\underline{\phi} ; \underline{\boldsymbol{\zeta}}, \underline{\psi}) \quad \forall \underline{\phi}, \underline{\boldsymbol{\zeta}}, \underline{\boldsymbol{\psi}} \in \mathbf{X},
$$

where $\mathcal{L}\left(\mathbf{X}, \mathbf{X}^{\prime}\right)$ is the set of linear and bounded operators from $\mathbf{X}$ to $\mathbf{X}^{\prime}$.

According to the above, we obtain that the Newton's method applied to problem (3.3) reads: Given $\underline{\phi}^{0} \in \mathbf{X}$, for $m \geq 1$, find $\underline{\phi}^{m} \in \mathbf{X}$, such that

$$
\left\langle\left(\mathcal{P}^{\prime}\left(\underline{\phi}^{m-1}\right)\right)\left(\underline{\phi}^{m}-\underline{\phi}^{m-1}\right), \underline{\psi}\right\rangle=-\left\langle\left(\mathcal{P}\left(\underline{\phi}^{m-1}\right)\right), \underline{\boldsymbol{\psi}}\right\rangle \quad \forall \underline{\boldsymbol{\psi}} \in \mathbf{X} .
$$

In what follows we apply Theorem 5.2 to prove the convergence of the iterative method (5.8), considering $\underline{\phi}^{0}=\underline{\mathbf{0}}$ as initial guess. This result is stated in the next theorem. 
Theorem 5.3 Assume that hypotheses of Theorem 3.4 hold and let $\underline{\phi} \in \mathbf{X}$ the unique solution of problem (3.3). Then, considering $\underline{\phi}^{0}=\underline{\mathbf{0}}$ as initial guess, the sequence $\left\{\underline{\phi}^{m}\right\}_{m \in \mathbb{N}}$ given by the Newton's method (5.8) converges to $\bar{\phi}$. In addition, There exist positive constants $K_{1}$ and $K_{2}$, independent of the solution and $m$, such that

$$
\left\|\underline{\phi}-\underline{\phi}^{m}\right\|_{\mathbf{X}} \leq \frac{1}{2^{m}}\left(2 K_{2}\right)^{2^{m}} \frac{K_{1}}{K_{2}}, \quad m \geq 0 .
$$

(Explicit constants $K_{1}$ and $K_{2}$ are provided in (5.12) and (5.14), respectively).

Proof. It suffices to verify that assumptions (1), (2) and (3) of Theorem 5.2 hold.

First, it is easy to see that

$$
\left\langle\mathcal{P}\left(\underline{\phi}^{0}\right), \underline{\boldsymbol{\psi}}\right\rangle=\langle\mathcal{P}(\underline{\mathbf{0}}), \underline{\boldsymbol{\psi}}\rangle=-\mathbf{F}(\underline{\boldsymbol{\psi}}) \leq\|\mathbf{f}\|_{0, \Omega}\left(\kappa_{1}^{2}+\nu^{2}\right)^{1 / 2}\|\underline{\boldsymbol{\psi}}\|_{\mathbf{X}} \quad \forall \underline{\boldsymbol{\psi}} \in \mathbf{X},
$$

from which

$$
\left\|\mathcal{P}\left(\underline{\phi}^{0}\right)\right\|_{\mathbf{X}^{\prime}} \leq\|\mathbf{f}\|_{0, \Omega}\left(\kappa_{1}^{2}+\nu^{2}\right)^{1 / 2}
$$

In turn, from Lemma 3.1, we have

$$
\left\langle\left(\mathcal{P}^{\prime}\left(\underline{\phi}^{0}\right)\right)(\underline{\boldsymbol{\psi}}), \underline{\boldsymbol{\psi}}\right\rangle=\mathbf{A}(\underline{\boldsymbol{\psi}}, \underline{\boldsymbol{\psi}}) \geq \alpha_{\mathbf{A}}\|\underline{\boldsymbol{\psi}}\|_{\mathbf{X}}^{2} \quad \forall \underline{\boldsymbol{\psi}} \in \mathbf{X}
$$

which implies that $\left[\mathcal{P}^{\prime}\left(\underline{\phi}^{0}\right)\right]^{-1}$ exists, and

$$
\left\|\left[\mathcal{P}^{\prime}\left(\underline{\phi}^{0}\right)\right]^{-1}\right\|_{\mathcal{L}\left(\mathbf{X}^{\prime}, \mathbf{X}\right)} \leq \frac{1}{\alpha_{\mathbf{A}}} .
$$

In this way, from (5.10) and (5.11) we obtain that assumption (1) and (2) hold with

$$
K_{1}=\frac{1}{\alpha_{\mathbf{A}}}\left(\kappa_{1}^{2}+\nu^{2}\right)^{1 / 2}\|\mathbf{f}\|_{0, \Omega} .
$$

Now, for (3) we first notice, after simple computations, that the second derivative of operator $\mathcal{P}$ is given by

$$
\left\langle\left(\left(\mathcal{P}^{\prime \prime}(\underline{\phi})(\underline{\varphi})\right)(\underline{\psi}), \underline{\boldsymbol{\zeta}}\right\rangle=\mathbf{C}(\underline{\varphi} ; \underline{\psi}, \underline{\zeta})+\mathbf{C}(\underline{\boldsymbol{\psi}} ; \underline{\varphi}, \underline{\boldsymbol{\zeta}}) \quad \forall \underline{\phi}, \underline{\varphi}, \underline{\psi}, \underline{\boldsymbol{\zeta}} \in \mathrm{X} .\right.
$$

It follows that

$$
\left\langle\left(\left(\mathcal{P}^{\prime \prime}(\underline{\phi})(\underline{\varphi})\right)(\underline{\psi}), \underline{\boldsymbol{\zeta}}\right\rangle \leq 2 C_{\mathbf{C}}\|\underline{\varphi}\|_{\mathbf{X}}\|\underline{\boldsymbol{\psi}}\|_{\mathbf{X}}\|\underline{\boldsymbol{\zeta}}\|_{\mathbf{X}} \quad \forall \underline{\phi}, \underline{\boldsymbol{\varphi}}, \underline{\boldsymbol{\psi}}, \underline{\boldsymbol{\zeta}} \in \mathbf{X},\right.
$$

which yields

$$
\left\|\mathcal{P}^{\prime \prime}(\underline{\phi})\right\|_{\mathcal{L}\left(\mathbf{X}, \mathcal{L}\left(\mathbf{X}, \mathbf{X}^{\prime}\right)\right)} \leq 2 C_{\mathbf{C}} .
$$

Hence, from (5.11) and (5.13) we get that assumption (3) holds with

$$
K_{2}=\frac{2 C_{\mathbf{C}}}{\alpha_{\mathbf{A}}} .
$$

Therefore, from (3.17), (5.12) and (5.14) we finally get

$$
K_{3}:=K_{1} K_{2}=\frac{2 C_{\mathbf{C}}\left(\kappa_{1}^{2}+\nu^{2}\right)^{1 / 2}}{\alpha_{\mathbf{A}}^{2}}\|\mathbf{f}\|_{0, \Omega}<\frac{1}{2},
$$

which concludes the proof. 
Remark 5.4 We observe that, according to the definition of operator $\mathcal{P}$ in (5.6), the Newton's method described in (5.8) can be rewritten in terms of the forms $\mathbf{A}$ and $\mathbf{C}$ as follows: Find $\underline{\phi}^{m} \in \mathbf{X}$ such that

$$
\mathbf{A}\left(\underline{\phi}^{m}, \underline{\boldsymbol{\psi}}\right)+\mathbf{C}\left(\underline{\phi}^{m} ; \underline{\phi}^{m-1}, \underline{\boldsymbol{\psi}}\right)+\mathbf{C}\left(\underline{\phi}^{m-1} ; \underline{\boldsymbol{\phi}}^{m}, \underline{\boldsymbol{\psi}}\right)=\mathbf{C}\left(\underline{\phi}^{m-1} ; \underline{\phi}^{m-1}, \underline{\boldsymbol{\psi}}\right)+\mathbf{F}(\underline{\boldsymbol{\psi}}),
$$

for all $\boldsymbol{\psi} \in \mathbf{X}$.

\section{$6 \quad$ Numerical results}

In this section we present three examples illustrating the performance of our augmented mixed finite element scheme (4.4) on a set of uniform triangulations of the corresponding domains and considering the finite element spaces introduced in Section 4.5. Our implementation is based on a FreeFem ++ code (see [26]), in conjunction with the direct linear solver UMFPACK (see [10]). Regarding the implementation of the iterative methods, the iterations are terminated once the relative error of the entire coefficient vectors between two consecutive iterates is sufficiently small, i.e.,

$$
\frac{\| \text { coeff }^{m+1}-\text { coeff }^{m} \|_{l^{2}}}{\left\|\operatorname{coeff}^{m+1}\right\|_{l^{2}}} \leq t o l,
$$

where $\|\cdot\|_{l^{2}}$ is the standard $l^{2}$-norm in $\mathbb{R}^{N}$, with $N$ denoting the total number of degrees of freedom defining the finite element subspaces $\mathbb{H}_{h}$ and $\mathbf{H}_{h}$, and tol is a fixed tolerance to be specified on each example. As suggested in Theorem 5.3, for each example shown below we simply take $\underline{\phi}^{0}:=\left(\mathbf{T}_{h, 0}^{0}, \mathbf{u}_{h}^{0}\right)=(\mathbf{0}, \mathbf{0})$ as initial guess.

We now introduce some additional notations. The individual errors are denoted by:

$$
\begin{aligned}
& \mathrm{e}(\mathbf{u}):=\left\|\mathbf{u}-\mathbf{u}_{h}\right\|_{1, \Omega}, \quad \mathrm{e}\left(\mathbf{T}_{0}\right):=\left\|\mathbf{T}_{0}-\mathbf{T}_{h, 0}\right\|_{\operatorname{div}, \Omega}, \quad \mathrm{e}(p):=\left\|p-p_{h}\right\|_{0, \Omega}, \\
& \mathrm{e}(\boldsymbol{\omega}):=\left\|\boldsymbol{\omega}-\boldsymbol{\omega}_{h}\right\|_{0, \Omega}, \quad \mathrm{e}(\nabla \mathbf{u}):=\left\|\nabla \mathbf{u}-\mathbf{G}_{h}\right\|_{0, \Omega}, \quad \mathrm{e}(\tilde{\boldsymbol{\sigma}}):=\left\|\tilde{\boldsymbol{\sigma}}-\tilde{\boldsymbol{\sigma}}_{h}\right\|_{0, \Omega} .
\end{aligned}
$$

where $p_{h}, \boldsymbol{\omega}_{h}, \mathbf{G}_{h}$, and $\tilde{\boldsymbol{\sigma}}_{h}$ are the variables computed by the prostprocess (4.18). Also, we let $r\left(\mathbf{T}_{0}\right), r(\mathbf{u}), r(p), r(\boldsymbol{\omega}), r(\nabla \mathbf{u})$, and $r(\tilde{\boldsymbol{\sigma}})$ be the experimental rates of convergence given by

$$
\begin{aligned}
& r\left(\mathbf{T}_{0}\right):=\frac{\log \left(\mathrm{e}\left(\mathbf{T}_{0}\right) / \mathrm{e}^{\prime}\left(\mathbf{T}_{0}\right)\right)}{\log \left(h / h^{\prime}\right)}, \quad r(\mathbf{u}):=\frac{\log \left(\mathrm{e}(\mathbf{u}) / \mathrm{e}^{\prime}(\mathbf{u})\right)}{\log \left(h / h^{\prime}\right)}, \quad r(p):=\frac{\log \left(\mathrm{e}(p) / \mathrm{e}^{\prime}(p)\right)}{\log \left(h / h^{\prime}\right)}, \\
& r(\boldsymbol{\omega}):=\frac{\log \left(\mathrm{e}(\boldsymbol{\omega}) / \mathrm{e}^{\prime}(\boldsymbol{\omega})\right)}{\log \left(h / h^{\prime}\right)}, \quad r(\nabla \mathbf{u}):=\frac{\log \left(\mathrm{e}(\nabla \mathbf{u}) / \mathrm{e}^{\prime}(\nabla \mathbf{u})\right)}{\log \left(h / h^{\prime}\right)}, \quad r(\tilde{\boldsymbol{\sigma}}):=\frac{\log \left(\mathrm{e}(\tilde{\boldsymbol{\sigma}}) / \mathrm{e}^{\prime}(\tilde{\boldsymbol{\sigma}})\right)}{\log \left(h / h^{\prime}\right)},
\end{aligned}
$$

where $h$ and $h^{\prime}$ denote two consecutive meshsizes with errors e and $\mathrm{e}^{\prime}$.

Our first example is carried out in order to assess the influence of the parameters $\kappa_{1}, \kappa_{2}$ and $\kappa_{3}$ on the performance of our augmented mixed formulation (4.4). For this example we consider the domain $\Omega:=(-1,1)^{2}$, the viscosity constant $\nu=1$, and take $\mathbf{f}$ so that the exact solution is given by

$$
\begin{aligned}
& \mathbf{u}\left(x_{1}, x_{2}\right)=\left(\begin{array}{c}
\pi \sin \left(\pi x_{1}\right)^{2} \cos \left(\pi x_{2}\right) \\
-2 \pi \cos \left(\pi x_{1}\right) \sin \left(\pi x_{1}\right) \sin \left(\pi x_{2}\right)
\end{array}\right), \\
& p\left(x_{1}, x_{2}\right)=5 x_{1} \sin \left(x_{2}\right)
\end{aligned}
$$



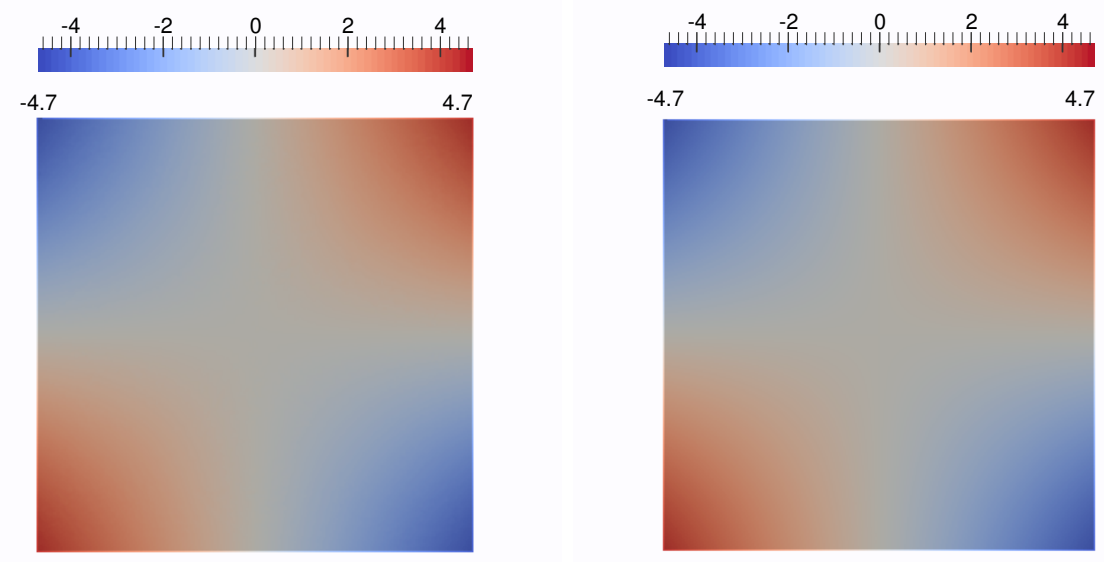

Figure 6.1: Example 1: $p_{h}$ (left) and $p$ (right) with $N=157339$

In order to emphasize the robustness of (4.4) with respect to the parameters $\kappa_{1}, \kappa_{2}$ and $\kappa_{3}$, as suggested in Remark 3.2, we choose $\left(\kappa_{1}, \kappa_{2}, \kappa_{3}\right)=\left(\nu^{2}, \nu, \frac{\nu^{2}}{2}\right)$ and $\left(\kappa_{1}, \kappa_{2}, \kappa_{3}\right)=\left(\frac{3 \nu^{2}}{4}, \frac{3 \nu}{2}, \frac{3 \nu^{2}}{8}\right)$, which, certainly, satisfy the assumptions of Lemma 3.1. In Tables 6.1 and 6.2 we summarize the convergence history for a sequence of uniform triangulations, considering the finite element spaces introduced in Section 4.5 with $k=0$ and $k=1$, and solving the nonlinear problem with the Newton's method defined in (5.15). We observe there that the rate of convergence $O\left(h^{k+1}\right)$ predicted by Theorem 4.8 (when $s=k+1$ ) is attained in all the cases. In addition, the specific results displayed in Tables 6.1 and 6.2 , showing almost no difference between $\left(\kappa_{1}, \kappa_{2}, \kappa_{3}\right)=$ $\left(\nu^{2}, \nu, \frac{\nu^{2}}{2}\right)$ and $\left(\kappa_{1}, \kappa_{2}, \kappa_{3}\right)=\left(\frac{3 \nu^{2}}{4}, \frac{3 \nu}{2}, \frac{3 \nu^{2}}{8}\right)$ illustrate the robustness of scheme (4.4), with respect to the choice of these parameters. Next, in Figure 6.1 we display the approximate pressure (to the left) obtained from the $\mathrm{RT}_{0}-\mathrm{P}_{1}$ approximation with $N=157339$ degrees of freedom, and we compare it with the exact one (to the right). In addition, in Figure 6.2 we display the magnitude and vector field of the approximate velocity (to the left) and we compare it with the exact velocity (to the right). In both cases we observe that the finite element subspaces employed provide very accurate approximations to the unknowns.

Our second example focusses on the performance of the iterative methods as a function of the viscosity $\nu$, considering the analytical solution $(\mathbf{u}, p)$ obtained by Kovasznay in [31]. For the domain $\Omega:=(-1 / 2,3 / 2) \times(0,2)$ and for a given $\nu$, this solution is given by

$$
\begin{aligned}
& \mathbf{u}\left(x_{1}, x_{2}\right)=\left(\begin{array}{c}
1-e^{\lambda x_{1}} \cos \left(2 \pi x_{2}\right) \\
\frac{\lambda}{2 \pi} e^{\lambda x_{1}} \sin \left(2 \pi x_{2}\right)
\end{array}\right), \\
& p\left(x_{1}, x_{2}\right)=-\frac{1}{2} e^{2 \lambda x_{1}}+\bar{p},
\end{aligned}
$$


Errors and rates of convergence for the mixed $\mathrm{RT}_{0}-\mathrm{P}_{1}$ approximation

\begin{tabular}{c|c||c|c||c|c||c|c}
$N$ & $h$ & $\mathrm{e}\left(\mathbf{T}_{0}\right)$ & $r\left(\mathbf{T}_{0}\right)$ & $\mathrm{e}(\mathbf{u})$ & $r(\mathbf{u})$ & $\mathrm{e}(p)$ & $r(p)$ \\
\hline 691 & 0.4129 & 90.2251 & - & 14.0226 & - & 4.8682 & - \\
2595 & 0.1901 & 47.6284 & 0.8235 & 7.0122 & 0.8933 & 2.7057 & 0.7571 \\
9955 & 0.0968 & 23.9312 & 1.0192 & 3.6224 & 0.9781 & 1.4606 & 0.9130 \\
39195 & 0.0527 & 12.0739 & 1.1269 & 1.8405 & 1.1154 & 0.7152 & 1.1762 \\
157339 & 0.0307 & 5.9814 & 1.2960 & 0.9051 & 1.3095 & 0.3541 & 1.2970
\end{tabular}

\begin{tabular}{c|c||c|c||c|c||c|c||c}
$N$ & $h$ & $\mathrm{e}(\boldsymbol{\omega})$ & $r(\boldsymbol{\omega})$ & $\mathrm{e}(\nabla \mathbf{u})$ & $r(\nabla \mathbf{u})$ & $\mathrm{e}(\tilde{\boldsymbol{\sigma}})$ & $r(\tilde{\boldsymbol{\sigma}})$ & Iterations \\
\hline 691 & 0.4129 & 6.2193 & - & 14.069 & - & 23.0156 & - & 5 \\
2595 & 0.1901 & 2.6606 & 1.0944 & 6.6236 & 0.9710 & 11.5540 & 0.8883 & 4 \\
9955 & 0.0968 & 1.2866 & 0.9580 & 3.2792 & 1.0411 & 5.8339 & 1.0120 & 4 \\
39195 & 0.0527 & 0.5917 & 1.2796 & 1.5895 & 1.1929 & 2.8858 & 1.1595 & 4 \\
157339 & 0.0307 & 0.2887 & 1.3238 & 0.7799 & 1.3138 & 1.4201 & 1.3083 & 4
\end{tabular}

Errors and rates of convergence for the mixed $\mathrm{RT}_{1}-\mathrm{P}_{2}$ approximation

\begin{tabular}{c|c||c|c||c|c||c|c}
$N$ & $h$ & $\mathrm{e}\left(\mathbf{T}_{0}\right)$ & $r\left(\mathbf{T}_{0}\right)$ & $\mathrm{e}(\mathbf{u})$ & $r(\mathbf{u})$ & $\mathrm{e}(p)$ & $r(p)$ \\
\hline 2315 & 0.4129 & 23.6263 & - & 3.3517 & - & 1.9654 & - \\
8883 & 0.1901 & 5.7915 & 1.8122 & 0.8857 & 1.7154 & 0.5413 & 1.6619 \\
34451 & 0.0968 & 1.5250 & 1.9760 & 0.2188 & 2.0702 & 0.1455 & 1.9456 \\
136407 & 0.0527 & 0.3865 & 2.2611 & 0.0566 & 2.2270 & 0.0397 & 2.1386 \\
549143 & 0.0307 & 0.0939 & 2.6103 & 0.0139 & 2.5957 & 0.0098 & 2.5783
\end{tabular}

\begin{tabular}{c|c||c|c||c|c||c|c||c}
$N$ & $h$ & $\mathrm{e}(\boldsymbol{\omega})$ & $r(\boldsymbol{\omega})$ & $\mathrm{e}(\nabla \mathbf{u})$ & $r(\nabla \mathbf{u})$ & $\mathrm{e}(\tilde{\boldsymbol{\sigma}})$ & $r(\tilde{\boldsymbol{\sigma}})$ & Iterations \\
\hline 231 & 0.4129 & 3.3323 & - & 7.0599 & - & 10.8747 & - & 4 \\
8883 & 0.1901 & 0.9016 & 1.6849 & 1.9217 & 1.6772 & 2.9757 & 1.6704 & 4 \\
34451 & 0.0968 & 0.2165 & 2.1123 & 0.4934 & 2.0135 & 0.8006 & 1.9442 & 4 \\
136407 & 0.0527 & 0.0551 & 2.2520 & 0.1270 & 2.2330 & 0.2087 & 2.2144 & 4 \\
549143 & 0.0307 & 0.0133 & 2.6231 & 0.0300 & 2.6060 & 0.0512 & 2.5946 & 4
\end{tabular}

Table 6.1: Example 1: Degrees of freedom, meshsizes, errors, rates of convergence and number of iterations for the mixed $\mathrm{RT}_{0}-\mathrm{P}_{1}$ and $\mathrm{RT}_{1}-\mathrm{P}_{2}$ approximations of the Navier-Stokes problem with $\left(\kappa_{1}, \kappa_{2}, \kappa_{3}\right)=\left(\nu^{2}, \nu, \frac{\nu^{2}}{2}\right)$. 
Errors and rates of convergence for the mixed $\mathrm{RT}_{0}-\mathrm{P}_{1}$ approximation

\begin{tabular}{c|c||c|c||c|c||c|c}
$N$ & $h$ & $\mathrm{e}\left(\mathbf{T}_{0}\right)$ & $r\left(\mathbf{T}_{0}\right)$ & $\mathrm{e}(\mathbf{u})$ & $r(\mathbf{u})$ & $\mathrm{e}(p)$ & $r(p)$ \\
\hline 691 & 0.4129 & 90.2374 & - & 13.9627 & - & 4.7906 & - \\
2595 & 0.1901 & 47.6335 & 0.8235 & 7.0124 & 0.8877 & 2.7074 & 0.7356 \\
9955 & 0.0968 & 23.9321 & 1.0193 & 3.6227 & 0.9781 & 1.4593 & 0.9152 \\
39195 & 0.0527 & 12.0741 & 1.1270 & 1.8405 & 1.1155 & 0.7155 & 1.1741 \\
157339 & 0.0307 & 5.9814 & 1.2960 & 0.9051 & 1.3095 & 0.3541 & 1.2978
\end{tabular}

\begin{tabular}{c|c||c|c||c|c||c|c||c}
$N$ & $h$ & $\mathrm{e}(\boldsymbol{\omega})$ & $r(\boldsymbol{\omega})$ & $\mathrm{e}(\nabla \mathbf{u})$ & $r(\nabla \mathbf{u})$ & $\mathrm{e}(\tilde{\boldsymbol{\sigma}})$ & $r(\tilde{\boldsymbol{\sigma}})$ & Iterations \\
\hline 691 & 0.4129 & 6.2267 & - & 13.9904 & - & 22.7738 & - & 4 \\
2595 & 0.1901 & 2.6626 & 1.0950 & 6.5992 & 0.9685 & 11.4952 & 0.8812 & 4 \\
9955 & 0.0968 & 1.2869 & 1.0768 & 3.2748 & 1.0377 & 5.8230 & 1.0072 & 4 \\
39195 & 0.0527 & 0.5917 & 1.2797 & 1.5890 & 1.1912 & 2.8848 & 1.1570 & 4 \\
157339 & 0.0307 & 0.2887 & 1.3239 & 0.7798 & 1.3134 & 1.4199 & 1.3079 & 4
\end{tabular}

Errors and rates of convergence for the mixed $\mathrm{RT}_{1}-\mathrm{P}_{2}$ approximation

\begin{tabular}{c|c||c|c||c|c||c|c}
$N$ & $h$ & $\mathrm{e}\left(\mathbf{T}_{0}\right)$ & $r\left(\mathbf{T}_{0}\right)$ & $\mathrm{e}(\mathbf{u})$ & $r(\mathbf{u})$ & $\mathrm{e}(p)$ & $r(p)$ \\
\hline 2315 & 0.4129 & 23.6269 & - & 3.3457 & - & 1.9558 & - \\
8883 & 0.1901 & 5.7915 & 1.8122 & 0.8853 & 1.7137 & 0.5402 & 1.6584 \\
34451 & 0.0968 & 1.5250 & 1.9760 & 0.2188 & 2.0697 & 0.1454 & 1.9435 \\
136407 & 0.0527 & 0.3865 & 2.2611 & 0.0566 & 2.2269 & 0.0397 & 2.1377 \\
549143 & 0.0307 & 0.0939 & 2.6103 & 0.0139 & 2.5957 & 0.0098 & 2.5780
\end{tabular}

\begin{tabular}{c|c||c|c||c|c||c|c||c}
$N$ & $h$ & $\mathrm{e}(\boldsymbol{\omega})$ & $r(\boldsymbol{\omega})$ & $\mathrm{e}(\nabla \mathbf{u})$ & $r(\nabla \mathbf{u})$ & $\mathrm{e}(\tilde{\boldsymbol{\sigma}})$ & $r(\tilde{\boldsymbol{\sigma}})$ & Iterations \\
\hline 231 & 0.4129 & 3.3322 & - & 7.0533 & - & 10.8542 & - & 4 \\
8883 & 0.1901 & 0.9016 & 1.6849 & 1.9210 & 1.6765 & 2.9733 & 1.6690 & 4 \\
34451 & 0.0968 & 0.2165 & 2.1124 & 0.4933 & 2.0132 & 0.8003 & 1.9435 & 4 \\
136407 & 0.0527 & 0.0551 & 2.2540 & 0.1272 & 2.2327 & 0.2087 & 2.2140 & 4 \\
549143 & 0.0307 & 0.0133 & 2.6231 & 0.0310 & 2.6060 & 0.0512 & 2.5945 & 4
\end{tabular}

Table 6.2: ExAmple 1: Degrees of freedom, meshsizes, errors, rates of convergence and number of iterations for the mixed $\mathrm{RT}_{0}-\mathrm{P}_{1}$ and $\mathrm{RT}_{1}-\mathrm{P}_{2}$ approximations of the Navier-Stokes problem with $\left(\kappa_{1}, \kappa_{2}, \kappa_{3}\right)=\left(\frac{3 \nu^{2}}{4}, \frac{3 \nu}{2}, \frac{3 \nu^{2}}{8}\right)$. 

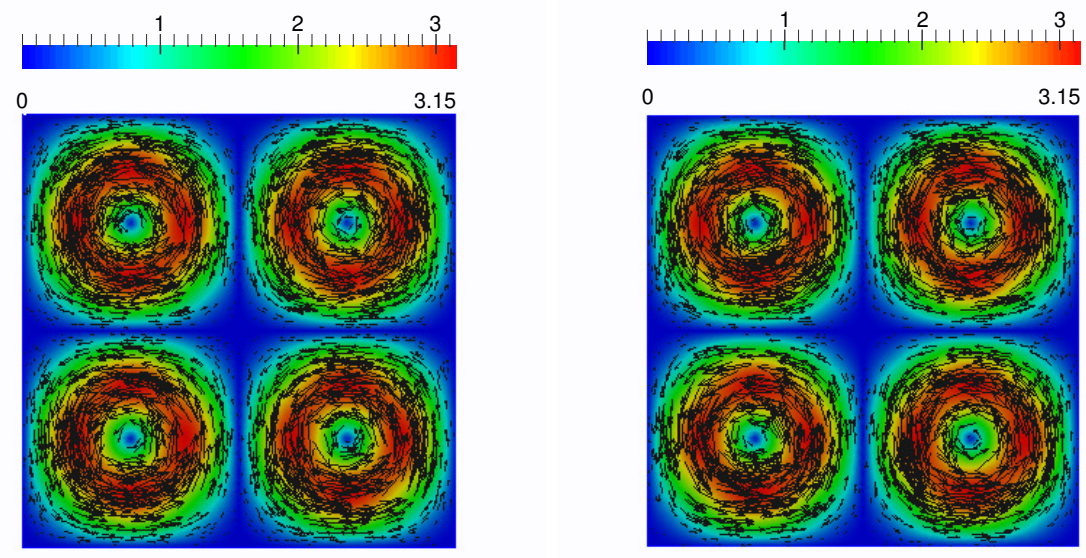

Figure 6.2: Example 1: velocitiy magnitudes $\left|\mathbf{u}_{h}\right|$ (left) and $|\mathbf{u}|$ (right) and velocity vector fields with $N=157339$

where

$$
\lambda:=\frac{-8 \pi^{2}}{\nu^{-1}+\sqrt{\nu^{-2}+16 \pi^{2}}},
$$

and the constant $\bar{p}$ is such that $\int_{\Omega} p=0$. Since, in this case the solution is inhomogeneous on the boundary, we need to modify the functional $\mathbf{F}$ as follows

$$
\mathbf{F}(\underline{\boldsymbol{\psi}}):=-\kappa_{1}(\mathbf{f}, \operatorname{div} \mathbf{S})_{\Omega}+\langle\mathbf{S n}, \mathbf{g}\rangle_{\Gamma}+\nu(\mathbf{f}, \mathbf{v})_{\Omega}+\kappa_{3}(\mathbf{g}, \mathbf{v})_{\Gamma} \quad \forall \underline{\boldsymbol{\psi}}=(\mathbf{S}, \mathbf{v}) \in \mathbf{X}
$$

where $\langle\cdot, \cdot\rangle_{\Gamma}$ denotes the duality pairing between $\mathbf{H}^{-1 / 2}(\Gamma)$ and $\mathbf{H}^{1 / 2}(\Gamma)$, and $\mathbf{g}:=\left.\mathbf{u}\right|_{\Gamma} \in \mathbf{H}^{1 / 2}(\Gamma)$.

In Table 6.3 we show the behaviour of the iterative methods (5.1) and (5.15) as a function of the viscosity number, considering different meshsizes and a tolerance $t o l=1 E-10$. The parameters $\kappa_{1}, \kappa_{2}, \kappa_{3}$ are simply chosen as $\left(\kappa_{1}, \kappa_{2}, \kappa_{3}\right)=(1, \nu, 1)$ and we consider the finite element spaces introduced in Section 4.5 with $k=0$. Here we observe that the smaller the parameter $\nu$ the higher the number of iterations. In addition we see that, as expected, the Newton's method is faster than the fixed-point iteration. Numerical experiments for smaller values of $\nu$ are not reported since both iterative methods need too many iterations to converge (more than 300). Next, the numerical results in Table 6.4 show the convergence history of the Newton's method (5.15) considering the viscosity $\nu=0.1$. We see there that the rate of convergence $O(h)$ provided by Theorem 4.8 and Corollary 4.9 for $s=1$ is attained by the unknowns and all the post-processed variables.

Finally, in our third example we assess the capability of a 3D implementation of our augmented scheme. Here, we consider the domain $\Omega:=(0,1)^{3}$, the viscosity constant $\nu=1$, the 
Number of iterations for the fixed-point method

\begin{tabular}{c||c|c|c|c|c}
\hline$\nu$ & $h=0.3801$ & $h=0.1901$ & $h=0.0982$ & $h=0.0530$ & $h=0.0266$ \\
\hline 1 & 15 & 15 & 13 & 11 & 10 \\
0.1 & 38 & 30 & 22 & 20 & 19 \\
0.059 & 59 & 59 & 24 & 21 & 20 \\
\hline
\end{tabular}

Number of iterations for the Newton's method

\begin{tabular}{c||c|c|c|c|c}
\hline$\nu$ & $h=0.3801$ & $h=0.1901$ & $h=0.0982$ & $h=0.0530$ & $h=0.0266$ \\
\hline 1 & 5 & 5 & 5 & 4 & 4 \\
0.1 & 7 & 6 & 6 & 5 & 5 \\
0.059 & 12 & 7 & 6 & 6 & 6 \\
\hline
\end{tabular}

Table 6.3: ExAmple 2: Convergence behavior of the iterative methods with respect to the parameter $\nu$.

\begin{tabular}{c|c||c|c||c|c||c|c}
$N$ & $h$ & $\mathrm{e}\left(\mathbf{T}_{0}\right)$ & $r\left(\mathbf{T}_{0}\right)$ & $\mathrm{e}(\mathbf{u})$ & $r(\mathbf{u})$ & $\mathrm{e}(p)$ & $r(p)$ \\
\hline 683 & 0.3801 & 5.1122 & - & 12.9236 & - & 2.6472 & - \\
2539 & 0.1901 & 3.0577 & 0.7415 & 6.7165 & 0.9442 & 1.2811 & 1.0471 \\
9923 & 0.0982 & 1.3439 & 1.2448 & 2.8092 & 1.3198 & 0.5635 & 1.2435 \\
39107 & 0.0530 & 0.5933 & 1.3248 & 1.1747 & 1.4129 & 0.2387 & 1.3919 \\
157051 & 0.0266 & 0.2908 & 1.0342 & 0.5554 & 1.0862 & 0.1159 & 1.0476 \\
$N$ & $h$ & $\mathrm{e}(\boldsymbol{\omega})$ & $r(\boldsymbol{\omega})$ & $\mathrm{e}(\nabla \mathbf{u})$ & $r(\nabla \mathbf{u})$ & $\mathrm{e}(\tilde{\boldsymbol{\sigma}})$ & $r(\tilde{\boldsymbol{\sigma}})$ \\
\hline 683 & 0.3801 & 21.9471 & - & 43.6479 & - & 8.9770 & - \\
2539 & 0.1901 & 13.7239 & 0.6773 & 20.2037 & 1.1113 & 3.9804 & 1.1733 \\
9923 & 0.0982 & 7.2897 & 0.9580 & 10.1070 & 1.0488 & 1.9127 & 1.1097 \\
39107 & 0.0530 & 3.4478 & 1.2133 & 4.7831 & 1.2123 & 0.8896 & 1.2405 \\
157051 & 0.0266 & 1.7457 & 0.9868 & 2.3881 & 1.0071 & 0.4405 & 1.0191
\end{tabular}

Table 6.4: Example 2: Degrees of freedom, meshsizes, errors, and rates of convergence for the mixed $\mathrm{RT}_{0}-\mathrm{P}_{1}$ approximation of the Navier-Stokes problem with $\nu=0.1$, utilizing the Newton's method. 


\begin{tabular}{c|c||c|c||c|c||c|c}
$N$ & $h$ & $\mathrm{e}\left(\mathbf{T}_{0}\right)$ & $r\left(\mathbf{T}_{0}\right)$ & $\mathrm{e}(\mathbf{u})$ & $r(\mathbf{u})$ & $\mathrm{e}(p)$ & $r(p)$ \\
\hline 442 & 0.7071 & 0.2621 & - & 0.0605 & - & 0.1578 & - \\
2968 & 0.3536 & 0.1400 & 0.9043 & 0.0286 & 1.0824 & 0.0794 & 0.9910 \\
21772 & 0.1768 & 0.0666 & 1.0730 & 0.0114 & 1.3210 & 0.0346 & 1.1991 \\
166804 & 0.0884 & 0.0313 & 1.0901 & 0.0045 & 1.3403 & 0.0151 & 1.2001 \\
1305892 & 0.0442 & 0.0151 & 1.0479 & 0.0019 & 1.2158 & 0.0070 & 1.1092
\end{tabular}

\begin{tabular}{c|c||c|c||c|c||c|c||c}
$N$ & $h$ & $\mathrm{e}(\boldsymbol{\omega})$ & $r(\boldsymbol{\omega})$ & $\mathrm{e}(\nabla \mathbf{u})$ & $r(\nabla \mathbf{u})$ & $\mathrm{e}(\tilde{\boldsymbol{\sigma}})$ & $r(\tilde{\boldsymbol{\sigma}})$ & Iterations \\
\hline 442 & 0.7071 & 0.0708 & - & 0.1259 & - & 0.3436 & - & 3 \\
2968 & 0.3536 & 0.0413 & 0.7766 & 0.0795 & 0.6628 & 0.1933 & 0.8298 & 3 \\
21772 & 0.1768 & 0.0225 & 0.8745 & 0.0449 & 0.8254 & 0.0980 & 0.9798 & 3 \\
166804 & 0.0884 & 0.0117 & 0.9431 & 0.0236 & 0.9254 & 0.0486 & 1.0121 & 3 \\
1305892 & 0.0442 & 0.0060 & 0.9748 & 0.0121 & 0.9697 & 0.0242 & 1.0059 & 3
\end{tabular}

Table 6.5: ExAmple 3: Degrees of freedom, meshsizes, errors, rates of convergence and number of iterations for the mixed $\mathrm{RT}_{0}-\mathrm{P}_{1}$ approximation of the $3 \mathrm{D}$ Navier-Stokes problem solved with the Newton's method.

parameters $\kappa_{1}=\nu^{2}, \kappa_{2}=\nu \kappa_{3}=\nu^{2} / 2$, and take $\mathbf{f}$ so that the exact solution is given by

$$
\begin{aligned}
& \mathbf{u}\left(x_{1}, x_{2}, x_{3}\right):=\left(\begin{array}{c}
4 x_{1}^{2} x_{2} x_{3}\left(x_{3}-1\right)\left(x_{2}-1\right)\left(x_{2}-x_{3}\right)\left(x_{1}-1\right)^{2} \\
-4 x_{1} x_{2}^{2} x_{3}\left(x_{2}-1\right)^{2}\left(x_{3}-1\right)\left(x_{1}-1\right)\left(x_{1}-x_{3}\right) \\
4 x_{1} x_{2} x_{3}^{2}\left(x_{3}-1\right)^{2}\left(x_{2}-1\right)\left(x_{1}-1\right)\left(x_{1}-x_{2}\right)
\end{array}\right), \\
& p\left(x_{1}, x_{2}, x_{3}\right):=x_{1}-\frac{1}{2}
\end{aligned}
$$

For this example we solve the nonlinear problem with the Newton's method defined in (5.15) with a tolerance $t o l=1 E-10$ and consider the finite element spaces introduced in Section 4.5 with $k=0$. In Table 6.5 we summarize the convergence history for a sequence of uniform triangulations. We observe there that the rate of convergence $O(h)$ predicted by Theorem 4.8 and Corollary 4.9 (when $s=1$ ) is attained in all the cases. Next, in Figure 6.3 we display the magnitude and vector field of the approximate velocity (top left and bottom left), and we compare them with their exact counterpart (top right and bottom right). Here, for the magnitude of the velocity we display the section of the cube below the plane $x_{1}+x_{2}+x_{3}=1.5$. In addition, in Figure 6.4 we display the second component of the approximate vorticity $\omega_{1, h}$ (top left) and the iso-surface of the approximate pressure, and we compare them with their exact counterpart (top right and bottom right). Again, for the vorticity we display the section of the cube below the plane $x_{1}+x_{2}+x_{3}=1.5$. All the graphics above were computed with $N=1305892$ degrees of freedom. We observe there that the finite element subspaces employed provide very accurate approximations to the unknowns.

Acknowledgments. The authors are grateful to Prof. Gabriel N. Gatica and Eligio Colmenares ( $\mathrm{CI}^{2} \mathrm{MA}$, Universidad de Concepción) for their helpful comments that helped us to improve the paper. 

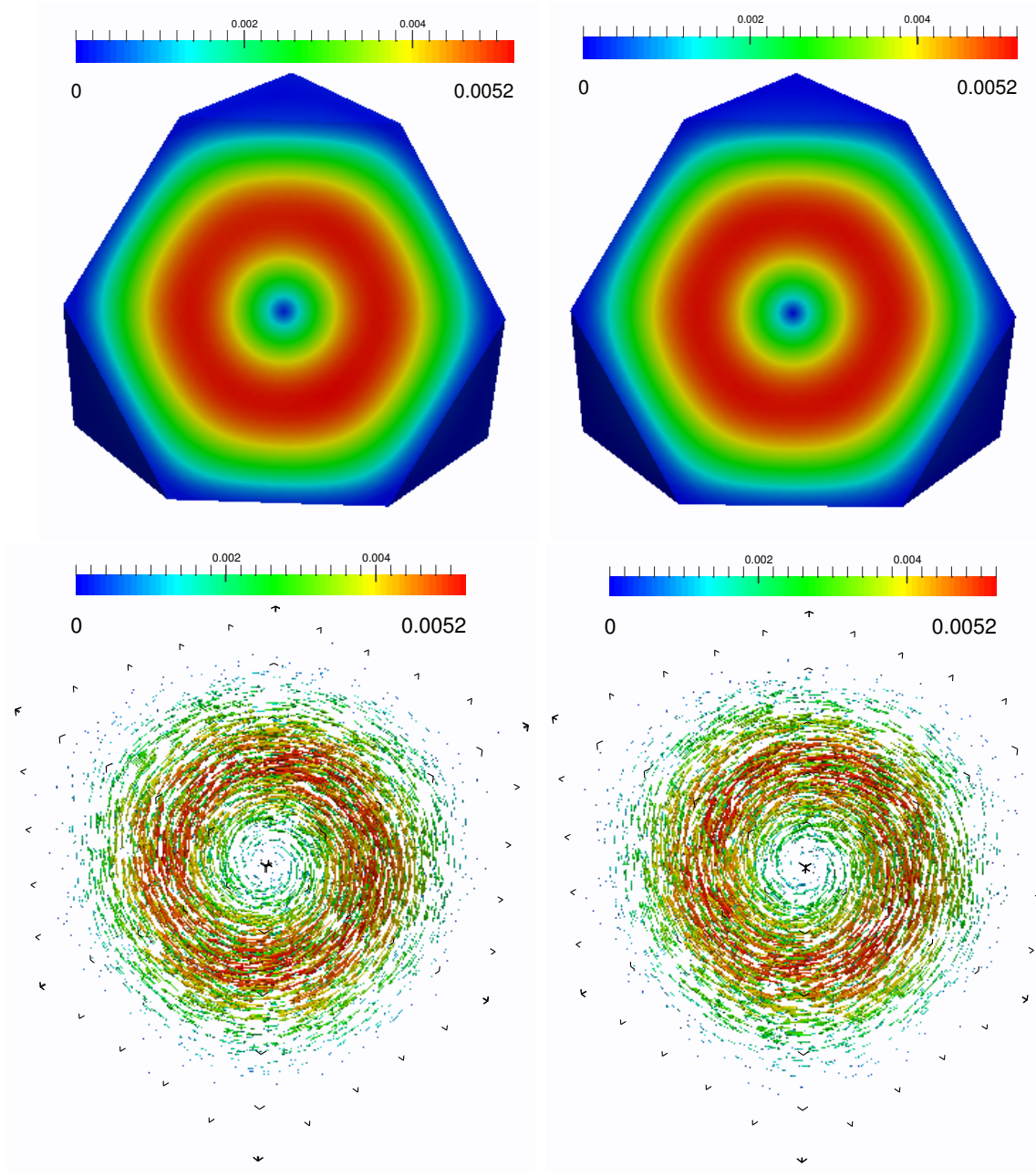

Figure 6.3: Example 3: velocity magnitudes $\left|\mathbf{u}_{h}\right|$ (top left) and $|\mathbf{u}|$ (top right), and velocity vector fields (approximate field to the left and exact field to the right) with $N=1305892$. 


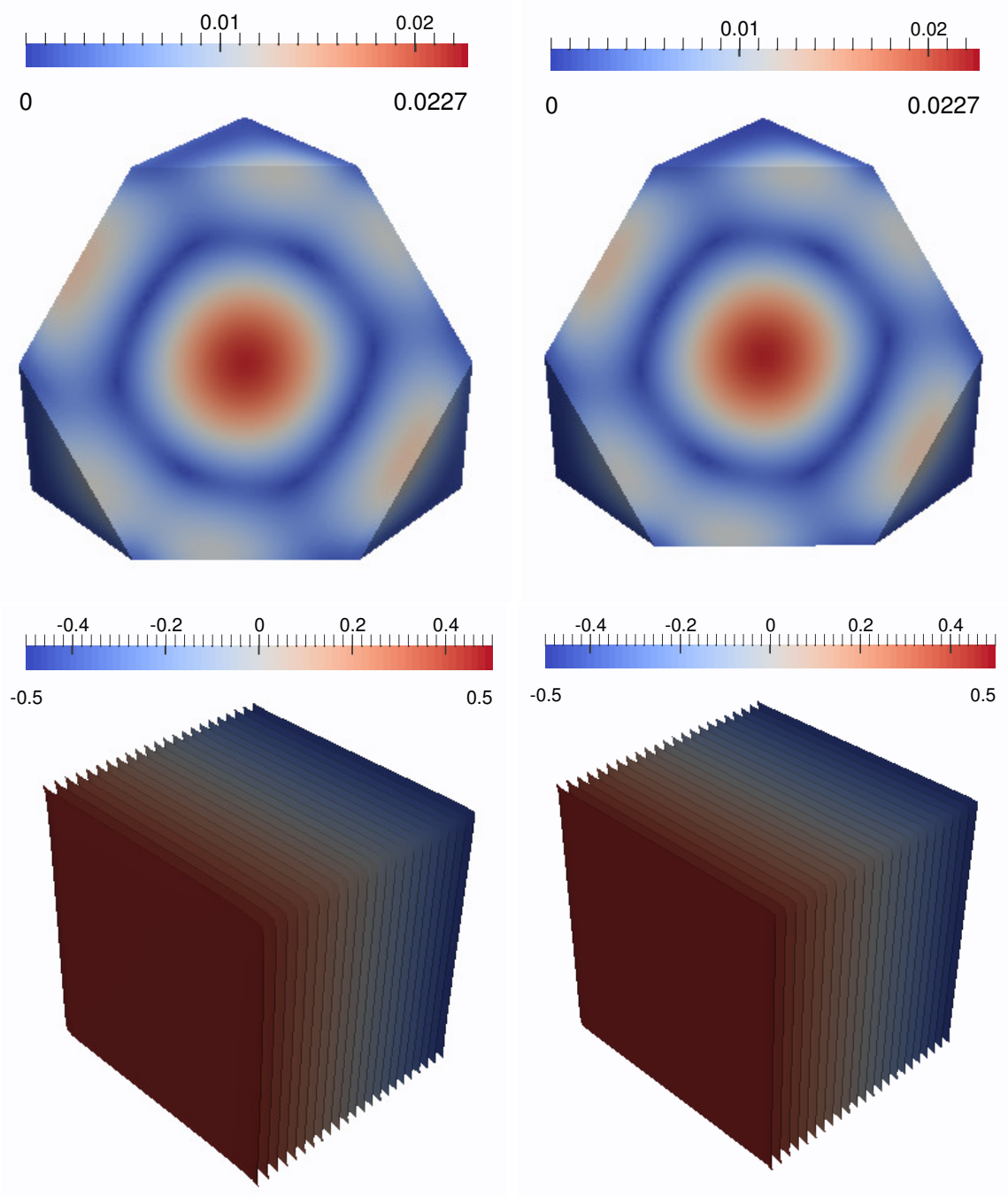

Figure 6.4: Example 3: second components of the vorticity $\omega_{2, h}$ (top left) and $\omega_{2}$ (top right), and iso-surface of the pressures $p_{h}$ (bottom left) and $p$ (bottom right) with $N=1305892$ 


\section{References}

[1] M. Alvarez, G.N. Gatica And R. Ruiz-Baier, An augmented mixed-primal finite element method for a coupled flow-transport problem. Preprint 2014-20, Centro de Investigación en Ingeniería Matemática (CI²MA), UDEC, (2014).

[2] D.N. Arnold, J. Douglas and Ch.P. Gupta, A family of higher order mixed finite element methods for plane elasticity. Numerische Mathematik, vol. 45, pp. 1-22, (1984).

[3] L. Badea, M. Discacciati And A. Quarteroni, Numerical analysis of the NavierStokes/Darcy coupling. Numerische Mathematik, vol. 115, no. 2, pp. 195-227 (2010).

[4] F. Brezzi And M. Fortin, Mixed and Hybrid Finite Element Methods. Springer Series in Computational Mathematics, 15. Springer-Verlag, New York, 1991.

[5] Z. Cai, Ch. Tong, P.S. Vassilevski and Ch. Wang, Mixed finite element methods for incompressible flow: stationary Stokes equations. Numerical Methods Partial Differential Equations, vol. 26, pp. 957-978, (2009).

[6] Z. CAi And Y. WAng, Pseudostress-velocity formulation for incompressible Navier-Stokes equations. International Journal for Numerical Methods in Fluids, 63, no. 3, 341-356, (2010).

[7] Z. Cai, Ch. Wang and S. Zhang, Mixed finite element methods for incompressible flow: stationary Navier-Stokes equations SIAM Journal on Numerical Analysis, vol 48, no. 1, 79-94, (2010).

[8] Z. Cai And S. Zhang, Mixed methods for stationary Navier-Stokes equations based on pseudostress-pressure-velocity formulation. Mathematics of Computation, vol. 81, no. 280, 1903-1927, (2012).

[9] P.G. Ciarlet, The finite Element Method for Elliptic Problems. North-Holland, Amsterdam, New York, Oxford, (1978).

[10] T. DAVIS, Algorithm 832: UMFPACK V4.3 - an unsymmetric-pattern multifrontal method. ACM Transactions on Mathematical Software, vol. 30, pp. 196-199, (2004).

[11] V.J. Ervin, J.S. Howell and I. Stanculescu, A dual-mixed approximation method for a three-eld model of a nonlinear generalized Stokes problem. Computer Methods in Applied Mechanics and Engineering, vol. 197, 33-40, pp. 2886-2900, (2008).

[12] M. Farhloul, S. Nicaise and L. Paquet, A refined mixed finite-element method for the stationary Navier-Stokes equations with mixed boundary conditions. IMA Journal of Numerical Analysis, vol. 28, no. 1, 25-45, (2008).

[13] M. Farhloul, S. Nicaise and L. Paquet, A priori and a posteriori error estimations for the dual mixed finite element method of the Navier-Stokes problem. Numerical Methods for Partial Differential Equations, vol. 25, no. 4, 843-869, (2009).

[14] L. Figueroa, G.N. Gatica and A. Márquez, Augmented mixed finite element methods for the stationary Stokes Equations. SIAM Journal on Scientific Computing, vol. 31, 2, pp. 1082-1119, (2008). 
[15] V. Girault and P.-A. Raviart, Finite Element Approximation of the Navier-Stokes Equations. Lecture Notes in Mathematics, 749. Springer-Verlag, Berlin-New York, (1979).

[16] G.N. Gatica, Analysis of a new augmented mixed finite element method for linear elasticity allowing $\mathrm{RT}_{0}-P_{1}-P_{0}$ approximations. ESAIM: Mathematical Modelling and Numerical Analysis, vol. 40, 1, pp. 1-28, (2006).

[17] G.N. GaticA, An augmented mixed finite element method for linear elasticity with nonhomogeneous Dirichlet conditions. Electronic Transactions on Numerical Analysis. vol. 26, pp. 421-438, (2007).

[18] G.N. Gatica, A Simple Introduction to the Mixed Finite Element Method. Theory and Applications. Springer Briefs in Mathematics, Springer, Cham Heidelberg New York Dordrecht London, (2014).

[19] G.N. Gatica, M. González and S. Meddahi, A low-order mixed finite element method for a class of quasi-Newtonian Stokes flows. I: A priori error analysis. Computer Methods in Applied Mechanics and Engineering, vol. 193, 9-11, pp. 881-892, (2004).

[20] G.N. Gatica, L.F. Gatica and A. Márquez, Augmented mixed finite element methods for a vorticity-based velocity-pressure-stress formulation of the Stokes problem in $2 D$. International Journal for Numerical Methods in Fluids, vol. 67, 4, pp. 450-477, (2011).

[21] G.N. Gatica, A. Márquez, R. Oyarzúa and R. Rebolledo, Analysis of an augmented fully-mixed approach for the coupling of quasi-Newtonian fluids and porous media. Computer Methods in Applied Mechanics and Engineering, vol. 270, 1, pp. 76-112, (2014).

[22] G.N. Gatica, A. Márquez and M.A. SÁnchez, Analysis of a velocity-pressurepseudostress formulation for incompressible flow. Computer Methods in Applied Mechanics and Engineering, vol. 199, 17-20, pp. 1064-1079, (2010).

[23] G.N. Gatica, A. Márquez And M.A. SÁnchez, A priori and a posteriori error analyses of a velocity-pseudostress formulation for a class of quasi-Newtonian Stokes flows. Computer Methods in Applied Mechanics and Engineering, vol. 200, 17-20, pp. 1619-1636, (2011).

[24] G.N. Gatica, R. Oyarzúa And F.J. Sayas, Analysis of fully-mixed finite element methods for the Stokes-Darcy coupled problem. Mathematics of Computation, vol. 80, 276, pp. 1911-1948, (2011).

[25] G.N. Gatica, R. Oyarzúa And F.J. Sayas, A twofold saddle point approach for the coupling of fluid flow with nonlinear porous media flow. IMA Journal of Numerical Analysis, vol. 32, 3, pp. 845-887, (2012).

[26] F. Hecht, New development in FreeFem++. Journal of Numerical Mathematics, vol. 20, 3-4, pp. 251-265, (2012).

[27] R. Hiptmain, Finite elements in computational electromagnetism. Acta Numerica, vol. 11, pp. 237-339, (2002). 
[28] J.S. Howell, Dual-mixed nite element approximation of Stokes and nonlinear Stokes problems using trace-free velocity gradients. Journal of Computational and Applied Mathematics, vol. 231, 2, pp. 780-792, (2009).

[29] J.S. Howell and N. Walkington, Dual Mixed Finite Element Methods for the Navier Stokes Equations. ESAIM: Mathematical Modelling and Numerical Analysis, vol. 47, 789$805,(2013)$.

[30] L. Kantorovich And G. Akilov, Functional analysis in normed spaces. Translated from the Russian by Brown, D.E., In:Robertson, A.P. (ed) International Series of Monographs in Pure and Applied Mathematics, vol. 46. The Macmillan Co., New York (1964).

[31] L. I. G. Kovasznay, Laminar flow behind a two-dimensional grid, Proceedings of the Cambridge Philosophical Society, vol. 44, pp. 58-62, (1948). 